Universidad

BIBLIOTECA

Document downloaded from the institutional repository of the University of Alcala: http://ebuah.uah.es/dspace/

This is a postprint version of the following published document:

Blasco, Ángel \& Pérez-Díaz, Sonia. 2019, "The limit point and the Tfunction", Journal of Symbolic Computation, vol. 94 (Sept-Oct. 2019), pp. 30-51

Available at https://doi.org/10.1016/i.jsc.2018.06.009

(C) 2019 Elsevier

(Article begins on next page)

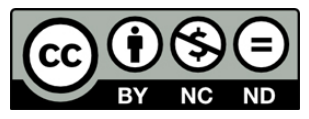

This work is licensed under a

Creative Commons Attribution-NonCommercial-NoDerivatives

4.0 International License. 


\title{
The Limit Point and the T-function
}

\author{
Angel Blasco and Sonia Pérez-Díaz \\ Departamento de Física y Matemáticas \\ Universidad de Alcalá \\ 28871-Alcalá de Henares, Madrid, Spain \\ angel.blasco@uah.es, sonia.perez@uah.es
}

\begin{abstract}
Let $\mathcal{P}(t) \in \mathbb{P}^{2}(\mathbb{K}(t))$ be a rational projective parametrization of a plane curve $\mathcal{C}$. In this paper, we introduce the notion of limit point, $P_{L}$, of $\mathcal{P}(t)$, and some remarkable properties of $P_{L}$ are obtained. In particular, if the singularities of $\mathcal{C}$ are $P_{1}, \ldots, P_{n}$ and $P_{L}$ (all of them ordinary) and their respective multiplicities are $m_{1}, \ldots, m_{n}$ and $m_{L}$, we show that $T(s)=$ $\prod_{i=1}^{n} H_{P_{i}}(s)^{m_{i}-1} H_{P_{L}}(s)^{m_{L}-1}$, where $T(s)$ is the univariate resultant of two polynomials obtained from $\mathcal{P}(t)$, and $H_{P_{1}}(s), \ldots, H_{P_{n}}(s), H_{P_{L}}(s)$ are the fibre functions of the singularities. The fibre function of a point $P$ is a polynomial $H_{P}(s)$ whose roots are the fibre of $P$. Thus, a complete classification of the singularities of a given plane curve, via the factorization of a resultant, is obtained.
\end{abstract}

Keywords: Algebraic Parametric Curve; Rational Parametrization; Singularities; Limit Point; T-function; Fibre Function.

\section{Introduction}

A given algebraic curve can be represented in different ways, such as implicitly by defining polynomials, parametrically by rational functions, or locally parametrically by power series expansions around a point. These representations all have their individual advantages: an implicit representation allows us to easily decide whether a given point lies on a given curve, a parametric representation allows us to generate points of a given curve over the desired coordinate fields, and using power series expansions one can, for instance, overcome the numerical problems of tracing a curve through a singularity. 
In the last years, important advances in the study and knowledge of a given algebraic variety (which is in general a curve or a surface) from its parametric representation has been obtained (see [7], [8], [16], etc.). In particular, an essential problem in computer aided geometric design (CAGD) is the detection of singularities (see e.g. [2], [3], [4], [12], [13], [14], [18] or [19]). Understanding the singularities of algebraic curves and surfaces is important for understanding their geometry. In fact, a difficult problem in CAGD is the handling of self-intersections, and the theory of singularities of algebraic varieties is potentially a tool for handling this problem. For instance, once the singularities are located, one can, use numerical methods to follow curve branches (see e.g. [9]).

In [12], several results in this sense are provided. In particular, some formulae for the computation of the multiplicity of a point are presented. These formulae simply involve the computation of the degree of a polynomial, directly determined from the parametrization. A further analysis on this topic can be found in [2] where, using the direct relation existing between the cardinality of the fibre of a given point and its multiplicity, it is shown how easily identify the singularities of the curve as those points whose fibre has more than one element. This topic is also addressed by some other authors although from a different point of view (see e.g. [1] and [3]).

In this paper, we observe that the relation between fibre and multiplicity fails for one point of the curve, the called limit point. Given a rational plane curve $\mathcal{C}$ over an algebraically closed field of characteristic zero $\mathbb{K}$, and a projective parametrization $\mathcal{P}(t) \in \mathbb{P}^{2}(\mathbb{K}(t))$ of degree $d$, the limit point is defined as $P_{L}:=\lim _{t \rightarrow \infty} \mathcal{P}(t) / t^{d}$. The point $P_{L}$ is on the curve, since $\mathcal{P}(t) \in \mathcal{C}$ for every $t \in \mathbb{K}$ and $\mathcal{C}$ is a closed set. However, it is not "well-represented" by the parametrization and in fact, its fibre is usually empty, i.e., there is no $t_{0} \in \mathbb{K}$ such that $\mathcal{P}\left(t_{0}\right)=P_{L}$. We say in this case that the limit point is unreachable via the parametrization $\mathcal{P}(t)$. This circumstance involves some difficulties, since the connection between fibre and multiplicity is lost and then, many results based on that connection do not hold.

Every rational parametrization has a limit point. If $P_{L}$ is not an affine point or it is a reachable affine point, $\mathcal{P}(t)$ is a normal parametrization. Otherwise, if $P_{L}$ is an affine point and it is not reachable via the parametrization, $\mathcal{P}(t)$ is not normal and $P_{L}$ is the critical point (see Subsection 6.3 in [16]). Thus, under a normal parametrization, every affine point of the curve is 
reachable via $\mathcal{P}(t)$. However, the problem persists, since $P_{L}$ remains a point of the curve (affine or at infinity) which is not "well-represented" by $\mathcal{P}(t)$ and, thus, its multiplicity is not the cardinality of its fibre. In fact, some important results presented in [2] and [12] hold for every point of the curve but for $P_{L}$. In particular, the main theorem in [2] (Theorem 3) holds only if $P_{L}$ is not a singularity.

The main goal in this paper is to explore the nature of the limit point and analyze the relation between its fibre and its multiplicity. As a remarkable result, we show that $P_{L}$ is reachable via $\mathcal{P}(t)$ only if it is a singular point of the given curve. In addition, we generalize Theorem 3 in [2] independently on whether the limit point is regular or not. In this way, we get a result that allows us to easily compute the ordinary singularities of a rational plane curve. Using the ideas presented in Section 4 in [2], one may easily generalize the results presented in this paper to the case of a given rational space curve in any dimension. Thus, a complete classification of the singularities of a given space curve, via the factorization of a resultant, is also obtained. A natural but more difficult problem is to consider the case of a given algebraic surface defined by a rational parametrization. In this case, similar results are expected to be provided. We will deal with this problem in a future work.

The structure of the paper is as follows. In Section 2, we provide the notation and some previous results. In Section 3 the notion of limit point, $P_{L}$, of the given parametrization $\mathcal{P}(t)$ is introduced and some important properties concerning the multiplicity of $P_{L}$ are obtained. We show these properties with some illustrative examples. In Section 4, we first summarize some previous properties concerning the $\mathrm{T}$-function introduced in [2]. In particular, Theorem 4.1 holds under the assumption that the limit point, $P_{L}$, is regular. The goal of Section 4 is to remove this condition and generalize the result to the case that $P_{L}$ is a singularity. The proof of the generalized theorem as well as a previous technical lemma appear in Section 5. These results are all illustrated with suitable examples.

\section{Notation and previous results}

Let $\mathcal{C}$ be a rational plane curve over an algebraically closed field of characteristic zero, $\mathbb{K}$, defined by the projective parametrization

$$
\mathcal{P}(t)=\left(p_{1}(t), p_{2}(t), p(t)\right) \in \mathbb{P}^{2}(\mathbb{K}(t)),
$$


where $\operatorname{gcd}\left(p_{1}, p_{2}, p\right)=1$. We assume that $\mathcal{C}$ is not a line. Let $d_{1}=\operatorname{deg}\left(p_{1}\right)$, $d_{2}=\operatorname{deg}\left(p_{2}\right), d_{3}=\operatorname{deg}(p)$ and $d=\max \left\{d_{1}, d_{2}, d_{3}\right\}$. Then, we write

$$
\left\{\begin{array}{l}
p_{1}(t)=a_{0}+a_{1} t+a_{2} t^{2}+\cdots+a_{d} t^{d} \\
p_{2}(t)=b_{0}+b_{1} t+b_{2} t^{2}+\cdots+b_{d} t^{d} \\
p(t)=c_{0}+c_{1} t+c_{2} t^{2}+\cdots+c_{d} t^{d} .
\end{array}\right.
$$

Note that, under these conditions, it holds that the degree of $\mathcal{C}$ is $d$ (see Theorem 6 in [12]).

Associated with $\mathcal{P}(t)$, we consider the induced rational map $\psi_{\mathcal{P}}: \mathbb{K} \longrightarrow$ $\mathcal{C} \subset \mathbb{P}^{2}(\mathbb{K}) ; t \longmapsto \mathcal{P}(t)$, and $\operatorname{deg}\left(\psi_{\mathcal{P}}\right)$ denotes the degree of the rational map $\psi_{\mathcal{P}}$ (see e.g. [6] pp. 80 or [17] pp. 143). We recall that the birationality of $\psi_{\mathcal{P}}$, i.e. the properness of $\mathcal{P}(t)$, is characterized by $\operatorname{deg}\left(\psi_{\mathcal{P}}\right)=1$ (see [6] and [17]). Furthermore, the degree of a rational map can be seen as the cardinality of the fibre of a generic element (see Theorem 7, pp. 76 in [17]). We will use this characterization in our reasoning. For this purpose, we denote by $\mathcal{F}_{\mathcal{P}}(P)$ the fibre of a point $P \in \mathcal{C}$ via the parametrization $\mathcal{P}(t)$; that is $\mathcal{F}_{\mathcal{P}}(P)=\mathcal{P}^{-1}(P)=\{t \in \mathbb{K} \mid \mathcal{P}(t)=P\}$.

It is well known that almost all points of $\mathcal{C}$ (except at most a finite number of points) are generated via $\mathcal{P}(t)$ by the same number of parameter values, and this number is $\operatorname{deg}\left(\psi_{\mathcal{P}}\right)$ (see Subsection 2.2. in [16]). Thus, intuitively speaking, $\operatorname{deg}\left(\psi_{\mathcal{P}}\right)$ measures the number of times that $\mathcal{P}(t)$ traces the curve when the parameter takes values in $\mathbb{K}$. In Chapter 4 in [16], it is proved that $\operatorname{deg}\left(\psi_{\mathcal{P}}\right)=\operatorname{deg}_{t}(G)$, where $G(s, t)=\operatorname{gcd}\left(G_{1}(s, t), G_{2}(s, t), G_{3}(s, t)\right)$ and

$$
\left\{\begin{array}{l}
G_{1}(s, t)=p_{1}(s) p(t)-p(s) p_{1}(t) \\
G_{2}(s, t)=p_{2}(s) p(t)-p(s) p_{2}(t) \\
G_{3}(s, t)=p_{1}(s) p_{2}(t)-p_{2}(s) p_{1}(t)
\end{array}\right.
$$

Observe that the parametrization is proper if and only if $\operatorname{deg}_{t}(G)=1$.

The cardinality of the fibre of $\psi_{\mathcal{P}}$ is the same for almost all points on $\mathcal{C}$; that is, all but finitely many points in $\mathcal{C}$ are generated, via $\mathcal{P}(t)$, by exactly $\operatorname{deg}_{t}(G)$ parameter values. Nevertheless, for finitely many exceptions, the cardinality may vary. We can compute the fibre of a particular point $P=(a: b: c) \in \mathbb{P}^{2}(\mathbb{K})$ by solving the corresponding fibre equations

$$
\left\{\begin{array}{l}
\phi_{1}(t):=a p(t)-c p_{1}(t)=0 \\
\phi_{2}(t):=b p(t)-c p_{2}(t)=0 \\
\phi_{3}(t):=a p_{2}(t)-b p_{1}(t)=0 .
\end{array}\right.
$$


Observe that $\mathcal{P}\left(t_{0}\right)=P$ if and only if $\phi_{1}\left(t_{0}\right)=\phi_{2}\left(t_{0}\right)=\phi_{3}\left(t_{0}\right)=0$. This motivates the following definition.

Definition 2.1. Let $P \in \mathbb{P}^{2}(\mathbb{K})$, and a parametrization $\mathcal{P}(t) \in \mathbb{P}^{2}(\mathbb{K}(t))$. We define the fibre function of $P$ via $\mathcal{P}(t)$ as $H_{P}(t):=\operatorname{gcd}\left(\phi_{1}, \phi_{2}, \phi_{3}\right)$.

Remark 2.2. Note that the roots of $H_{P}$ determine the fibre of $P$. In addition, we observe that the above expression for $H_{P}$ may be simplified if we consider the following cases (see Remark 2 in [2]):

- If $P$ is an affine point then $H_{P}(t)=\operatorname{gcd}\left(\phi_{1}(t), \phi_{2}(t)\right)$.

- If $P$ is a point at infinity then $H_{P}(t)=\operatorname{gcd}\left(p(t), \phi_{3}(t)\right)$.

Remark 2.3. Observe that if $P$ is a generic point of the input curve, $P=$ $(x: y: 1)$, it holds that, up to constants in $\mathbb{K}, \operatorname{Res}_{t}\left(\phi_{1}, \phi_{2}\right)=f(x, y)^{\operatorname{deg}\left(H_{P}\right)}$, where $f$ is the implicit polynomial defining the input curve and the resultant is the determinant of the Sylvester resultant (see e.g Subsection 4.5 in [16]). However, it holds that the determinant of the Bézout matrix is (up to a sign) the determinant of the Sylvester matrix (see e.g. [15]).

Throughout this paper we assume that $\mathcal{P}(t)$ is a proper parametrization (otherwise, we obtain a proper one by reparametrizing $\mathcal{P}$; see e.g. [11]). This means that $\operatorname{deg}\left(\psi_{\mathcal{P}}\right)=\operatorname{deg}_{t}(G)=1$ (see $\left.(1)\right)$ and, so, the cardinality of the fibre is 1 for almost every point of the curve. However, this cardinality may be different for finitely many points. In fact, the fibre of a singular point is greater than 1 (see e.g. [12]). The method proposed in [2] for computing the ordinary singularities of a rational curve is based on this idea. On the other hand, the cardinality of the fibre may be less than 1 if we consider the limit point of the parametrization.

Definition 2.4. We define the limit point of the parametrization $\mathcal{P}(t)$ as

$$
P_{L}=\lim _{t \rightarrow \infty} \mathcal{P}(t) / t^{d}=\left(a_{d}: b_{d}: c_{d}\right) .
$$

From this definition, it follows that every parametrization has only one limit point. In addition, $P_{L} \in \mathcal{C}$ since $\mathcal{P}(t) / t^{d} \in \mathcal{C}$ for every $t \in \mathbb{K}$ and $\mathcal{C}$ is a closed set. However, $P_{L}$ is not a conventional point. The following results, which are proved in [12], hold for every point of the curve but for $P_{L}$. 
Theorem 2.5. Let $\mathcal{C}$ be a rational algebraic curve defined by a proper parametrization $\mathcal{P}(t)$, with limit point $P_{L}$. Let $P \neq P_{L}$ be a point of $\mathcal{C}$ and let $H_{P}(t)=\prod_{i=1}^{n}\left(t-s_{i}\right)^{k_{i}}$ be its fibre function. Then, $\mathcal{C}$ has $n$ tangents at $P$ of multiplicities $k_{1}, \ldots, k_{n}$, respectively.

Corollary 2.6. Let $\mathcal{C}$ be a rational algebraic curve defined by a proper parametrization $\mathcal{P}(t)$, with limit point $P_{L}$. Let $P \neq P_{L}$ be a point of $\mathcal{C}$ and let $H_{P}(t)$ be its fibre function. Then, $\operatorname{mult}_{P}(\mathcal{C})=\operatorname{deg}\left(H_{P}\right)$.

Theorem 2.5 and Corollary 2.6 show that there exists a strong relation between the fibre of a point and its multiplicity, but they fail if the point is $P_{L}$ since this point is not "well-represented" by the parametrization. In fact, most of the times it holds that $\mathcal{F}_{\mathcal{P}}\left(P_{L}\right)=\emptyset$, i.e., there is no $t_{0} \in \mathbb{K}$ such that $\mathcal{P}\left(t_{0}\right)=P_{L}$. In this case, we say that the limit point is unreachable via the parametrization. In order to illustrate the concept of limit point, let us consider the ellipse defined by the projective parametrization

$$
\mathcal{P}(t)=\left(t^{2}-1, t^{2}-t, t^{2}+1\right) \in \mathbb{P}^{2}(\mathbb{C}(t))
$$

The limit point is, in this case, the affine point $P_{L}=(1,1)$. In Figure 1 (left), we plot the curve using $\mathcal{P}(t)$ with $-20 \leq t \leq 20$. In Figure 1 (right), we plot it using $\mathcal{P}(t)$ with $-60 \leq t \leq 60$. Note that $P_{L}$ is not reached by the parametrization but it would be reached in the limit, when $t$ tends to $\infty$.
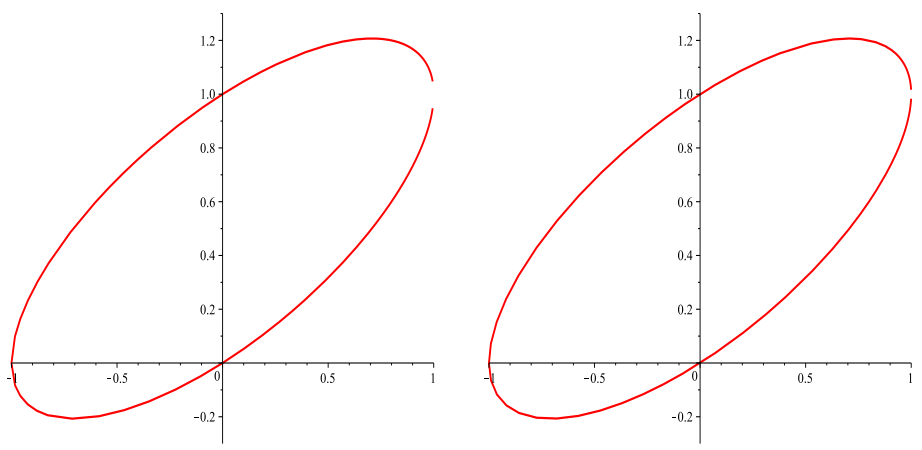

Figure 1: Curve $\mathcal{C}$ plotted from $\mathcal{P}(t)$ with $-20 \leq t \leq 20$ (left) and $-60 \leq t \leq 60$ (right)

We say that $\mathcal{P}(t)$ is a normal parametrization if $P_{L}$ is an infinity point or it is a reachable affine point; otherwise, $\mathcal{P}(t)$ is not normal and it is said that $P_{L}$ is the critical point (see Subsection 6.3 in [16]). 
In the next section some important properties as well as essential results concerning the limit point are obtained. In particular, we see that $P_{L}$ is a special point of $\mathcal{C}$ whose multiplicity has to be carefully computed. In addition, we prove that if the limit point is reached by $\mathcal{P}(t)$, then it is a singularity. These results will be used in Section 4, where the relation with the $T$-function is studied. The $\mathrm{T}$-function provides essential information about the singularities of the given curve $\mathcal{C}$. More precisely, its factorization provides the fibre functions of each singularity as well as its corresponding multiplicity (see [2]).

\section{The limit point and the hidden multiplicity}

In Definition 2.4, we introduce the notion of limit point of the parametrization $\mathcal{P}(t)$ as

$$
P_{L}=\lim _{t \rightarrow \infty} \mathcal{P}(t) / t^{d}=\left(a_{d}: b_{d}: c_{d}\right),
$$

where $d=\max \left\{d_{1}, d_{2}, d_{3}\right\}$, and $d_{1}=\operatorname{deg}\left(p_{1}\right), d_{2}=\operatorname{deg}\left(p_{2}\right), d_{3}=\operatorname{deg}(p)$. One may determine the fibre of $P_{L}$ from the corresponding fibre function, that we denote for this particular point as $H_{L}(t)$. That is,

$$
H_{L}(t):=\operatorname{gcd}\left(\phi_{1}^{L}, \phi_{2}^{L}, \phi_{3}^{L}\right),
$$

where

$$
\left\{\begin{aligned}
\phi_{1}^{L}(t) & =a_{d} p(t)-c_{d} p_{1}(t) \\
\phi_{2}^{L}(t) & =b_{d} p(t)-c_{d} p_{2}(t) \\
\phi_{3}^{L}(t) & =a_{d} p_{2}(t)-b_{d} p_{1}(t) .
\end{aligned}\right.
$$

The functions $\phi_{1}^{L}, \phi_{2}^{L}$ and $\phi_{3}^{L}$ are obtained as particular cases of those introduced in (2). Because of the importance of this point, we use a specific notation for them.

Remark 3.1. Remark 2.2 can be applied similarly for this special case (when the limit point is considered). More precisely, depending on whether $P_{L}$ is an affine point or an infinity point, its fibre function can be expressed as follows:

- If $P_{L}$ is an affine point, then $H_{L}(t)=\operatorname{gcd}\left(\phi_{1}^{L}(t), \phi_{2}^{L}(t)\right)$.

- If $P_{L}$ is a point at infinity, then $H_{L}(t)=\operatorname{gcd}\left(p(t), \phi_{3}^{L}(t)\right)$. 
Note that we can not compute the multiplicity of $P_{L}$ from the fibre since Corollary 2.6 does not hold in this particular case. Although $P_{L}$ is a point of $\mathcal{C}$, it is not "well-represented" by the parametrization $\mathcal{P}(t)$ and hence, the cardinality of the fibre does not provide its multiplicity. In the following, and throughout this section, we illustrate this statement and we present a method that allows to compute the multiplicity of $P_{L}$.

For this purpose, we consider a reparametrization of $\mathcal{P}(t), \mathcal{U}(t)$, such that $P_{L}$ is not the limit point (that is, $\mathcal{U}(t)$ has a limit point $U_{L} \neq P_{L}$ ). First, we assume that $P_{L}$ is not reachable via $\mathcal{P}(t)$, i.e., there is no $s_{0} \in \mathbb{K}$ such that $\mathcal{P}\left(s_{0}\right)=P_{L}$ (afterwards, we will analyze the case of $P_{L}$ reached by the parametrization $\mathcal{P}(t))$. In this case, $\mathcal{F}_{\mathcal{P}}\left(P_{L}\right)=\emptyset$ and the system in (3) does not have any solution. Then, we consider $\mathcal{U}(t)=\mathcal{P}(1 / t)$, and we write $\mathcal{U}(t)=\left(u_{1}(t), u_{2}(t), u(t)\right)$, where

$$
\left\{\begin{array}{l}
u_{1}(t)=p_{1}(1 / t) t^{d}=a_{0} t^{d}+a_{1} t^{d-1}+\cdots+a_{d} \\
u_{2}(t)=p_{2}(1 / t) t^{d}=b_{0} t^{d}+b_{1} t^{d-1}+\cdots+b_{d} \\
u(t)=p(1 / t) t^{d}=c_{0} t^{d}+c_{1} t^{d-1}+\cdots+c_{d}
\end{array}\right.
$$

The limit point of $\mathcal{U}(t)$ is

$$
U_{L}=\lim _{t \rightarrow \infty} \mathcal{U}(t) / t^{d}=\left(a_{0}: b_{0}: c_{0}\right)=\mathcal{P}(0)
$$

(note that $a_{0}=b_{0}=c_{0}=0$ is not possible since we are assuming that $\left.\operatorname{gcd}\left(p_{1}, p_{2}, p\right)=1\right)$. On the other hand, $P_{L}$ is a usual point of $\mathcal{C}$, which can be obtained as $P_{L}=\mathcal{U}(0)$. Since $\mathcal{U}(t)=\mathcal{P}(1 / t)$ and $\mathcal{P}(t)$ is proper, we get that $\mathcal{U}(t)$ is also a proper parametrization. Thus, we may apply Corollary 2.6 to determine the multiplicity of $P_{L}$ by computing the cardinality of $\mathcal{F}_{\mathcal{U}}\left(P_{L}\right)$.

In order to get it, we obtain the corresponding fibre function, that we denote as $H_{L}^{\mathcal{U}}(t)$. We observe that if $H_{L}^{\mathcal{U}}\left(s_{i}\right)=0$, for some $s_{i} \neq 0$, then $\mathcal{U}\left(s_{i}\right)=P_{L}$ and thus $\mathcal{P}\left(1 / s_{i}\right)=P_{L}$, which is impossible by assumption, since $P_{L}$ can not be reached by $\mathcal{P}(t)$. Thus, we have that $H_{L}^{\mathcal{U}}(t)=t^{r}$ for some $r \in \mathbb{N}$. Moreover, we have that $r \geq 1$, since $\mathcal{U}(0)=P_{L}$.

Therefore, from Corollary 2.6, we get that the multiplicity of $P_{L}$ is $r \geq 1$. We refer to this multiplicity (that can not be obtained from the parametrization $\mathcal{P}(t)$ ) as the hidden multiplicity of the limit point $P_{L}$, and we represent it as $m_{H}$. In the following, we illustrate the above procedure with an example. 
Example 3.2. Let $\mathcal{C}$ be a rational plane curve defined by the projective parametrization

$\mathcal{P}(t)=\left(t^{6}+2 t^{5}+2 t^{4}+3 t^{3}+2 t^{2}+t+1, t^{6}+2 t^{5}+t^{4}+t^{3}+t^{2}, t^{4}+t^{2}\right) \in \mathbb{P}^{2}(\mathbb{C}(t))$.

One may check that $\mathcal{P}$ is proper since $\operatorname{deg}_{t}(G)=1$ (see (1)). In addition, the limit point is $P_{L}=\lim _{t \rightarrow \infty} \mathcal{P}(t) / t^{6}=(1: 1: 0)$. In order to compute the fibre of $P_{L}$, we solve the system given in (3) that can be expressed as (see Remark 3.1)

$$
p(t)=t^{4}+t^{2}=0, \quad p_{1}(t)-p_{2}(t)=2 t^{3}+t+1=0 .
$$

This system does not have any solution, which implies that $P_{L}$ is not reached by the parametrization $\mathcal{P}(t)$. We consider the reparametrization

$\mathcal{U}(t)=\mathcal{P}(1 / t)=\left(t^{6}+t^{5}+2 t^{4}+3 t^{3}+2 t^{2}+2 t+1, t^{4}+t^{3}+t^{2}+2 t+1, t^{4}+t^{2}\right)$

and now $P_{L}$ is a usual point obtained as $P_{L}=\mathcal{U}(0)$. Furthermore, the fibre function is $H_{L}^{\mathcal{U}}(t)=t^{2}$ and thus, from Corollary 2.6, we conclude that $P_{L}$ is a singular point of $\mathcal{C}$ of multiplicity 2 (note that $m_{H}=2$ ).

Now, let us assume that the limit point, $P_{L}$, can be reached by the parametrization, i.e. $\mathcal{F}_{\mathcal{P}}\left(P_{L}\right) \neq \emptyset$. Then, $H_{L}(t)=\prod_{i=1}^{n}\left(t-s_{i}\right)^{k_{i}}$. If we could apply Corollary 2.6, we would get that the multiplicity of $P_{L}$ is $\operatorname{deg}\left(H_{L}\right)=k_{1}+\cdots+k_{n}$. However, this is not true since the fibre of $P_{L}$, via $\mathcal{P}(t)$, does not determine correctly the multiplicity of $P_{L}$.

In order to illustrate this statement, we reason as above, and we consider the reparametrization $\mathcal{U}(t)=\mathcal{P}(1 / t)$. Let us assume that $s_{i} \neq 0$ for $i=$ $1, \ldots, n$ (see Remark 3.4). Then, for each root $s_{i}$ of $H_{L}$ we have that $1 / s_{i}$ is a root of $H_{L}^{\mathcal{U}}$, since $\mathcal{U}\left(1 / s_{i}\right)=\mathcal{P}\left(s_{i}\right)=0$. In addition, $H_{L}^{\mathcal{U}}$ has one new root given by $t=0$, since $\mathcal{U}(0)=P_{L}$. Thus, from $H_{L}(t)=\prod_{i=1}^{n}\left(t-s_{i}\right)^{k_{i}}$, we easily get that

$$
H_{L}^{\mathcal{U}}(t)=t^{r} \prod_{i=1}^{n}\left(t-1 / s_{i}\right)^{k_{i}} .
$$

Finally, by applying Corollary 2.6 (note that $P_{L}$ is not the limit point of $\mathcal{U}(t))$, we conclude that

$$
\operatorname{mult}_{P_{L}}(\mathcal{C})=\operatorname{deg}\left(H_{L}^{\mathcal{U}}\right)=r+k_{1}+\cdots+k_{n} .
$$

Observe that part of this multiplicity is $k_{1}+\cdots+k_{n}=\operatorname{deg}\left(H_{L}\right)$. It is given by the degree of the fibre function $H_{L}(t)$, which is obtained from the 
parametrization $\mathcal{P}(t)$. We refer to this multiplicity as the visible multiplicity of the limit point $P_{L}$. However, there is another part, $r$, that could not be obtained via $\mathcal{P}(t)$; this is the hidden multiplicity $\left(m_{H}\right)$ introduced above. In the following definition, we summarize these notions.

Definition 3.3. Let $\mathcal{C}$ be a rational algebraic plane curve defined by a proper parametrization $\mathcal{P}(t)$ with limit point $P_{L}$. We denote $m_{L}=$ mult $_{P_{L}}(\mathcal{C})$, and we refer to $\operatorname{deg}\left(H_{L}\right)$ as the visible multiplicity of $P_{L}$. Then, $m_{L}=\operatorname{deg}\left(H_{L}\right)+$ $m_{H}$, where $m_{H}$ denotes the hidden multiplicity of $P_{L}$.

Remark 3.4. 1. The above reasoning is not correct if $s_{i}=0$ for some $i=$ $1, \ldots, n$, since $1 / s_{i}$ is not a root of the polynomial $H_{L}^{\mathcal{U}}(t)$. We observe that in this situation, $P_{L}$ is the limit point also with the parametrization $\mathcal{U}(t)$ (note that $U_{L}=\mathcal{P}(0)=P_{L}$ ) and thus, we can not apply Corollary 2.6. This problem can be solved by considering a new reparametrization of the form $\mathcal{Q}(t)=\mathcal{P}(\theta t /(t-1))$, where $\theta \neq 0$ and $\theta \neq s_{i}$ for every $i=1, \ldots, n$ (see Section 4). Reasoning with this reparametrization, we get an equivalent result.

2. Many authors (see e.g. [4]) consider the homogeneous parametrization $\overline{\mathcal{P}}(t, h)=\left(p_{1}(t / h) h^{d}, p_{2}(t / h) h^{d}, p(t / h) h^{d}\right)$. The multiplicity of $P_{L}$ is correctly obtained by using this parametrization. Observe that every point of $\mathcal{C}$ is reachable by $\overline{\mathcal{P}}$ since $\mathcal{P}(t)=\overline{\mathcal{P}}(t, 1)$ for every $t \in \mathbb{K}$, and $\overline{\mathcal{P}}(1, h)=\mathcal{U}(h)$ (which implies that $P_{L}=\overline{\mathcal{P}}(1,0)$ ). Therefore, $\overline{\mathcal{P}}(t, h)$ provides the visible and the hidden multiplicity of the limit point $P_{L}$. In fact, it is easy to check that the corresponding fibre function is

$$
\bar{H}_{L}(t, h)=h^{r} \prod_{i=1}^{n}\left(t-s_{i} h\right)^{k_{i}}
$$

where $\mathcal{P}\left(s_{i}\right)=P_{L}, i=1, \ldots, n, s_{i} \neq s_{j}, i \neq j, k_{1}+\cdots+k_{n}$ is the visible multiplicity and $r$ is the hidden one (see the analogy with (4)).

In the following proposition, we prove an important property concerning the limit point. Namely, if the limit point is reached by $\mathcal{P}(t)$, then it is a singularity. However, clearly the reciprocal is not true (see Example 3.2).

Proposition 3.5. If the limit point is reached by the parametrization $\mathcal{P}(t)$, then it is a singularity. 
Proof: The multiplicity of $P_{L}$ is given by

$$
\operatorname{mult}_{P_{L}}(\mathcal{C})=k_{1}+\cdots+k_{n}+m_{H},
$$

where $k_{1}+\cdots+k_{n}=\operatorname{deg}\left(H_{L}\right)$ is the visible multiplicity, and $m_{H}$ is the hidden multiplicity (see Definition 3.3). Note that $m_{H} \geq 1\left(\mathcal{U}(0)=P_{L}\right)$, and since $P_{L}$ is reached by the parametrization, then some of its multiplicity is visible, i.e. $k_{1}+\cdots+k_{n} \geq 1$. Therefore, $\operatorname{mult}_{P_{L}}(\mathcal{C}) \geq 2$ and thus, $P_{L}$ is a singularity.

Example 3.6. Let $\mathcal{C}$ be the rational curve defined over $\mathbb{C}$ by the projective parametrization $\mathcal{P}(t)=$

$\left(t^{6}+2 t^{5}+3 t^{4}+3 t^{2}+4 t^{3}+2 t+1,-t^{4}-t^{3}-t^{2}-2 t-1, t^{7}+3 t^{5}+t^{4}+3 t^{3}+t+2 t^{2}+1\right)$.

One may check that $\mathcal{P}$ is proper since $\operatorname{deg}_{t}(G)=1$ (see (1)). In addition, the limit point is $P_{L}=\lim _{t \rightarrow \infty} \mathcal{P}(t) / t^{7}=(0: 0: 1)$. The fibre of $P_{L}$ is obtained by solving the system in (3), that can be expressed as (see Remark 3.1)

$$
\left\{\begin{array}{l}
p_{1}(t)=t^{6}+2 t^{5}+3 t^{4}+3 t^{2}+4 t^{3}+2 t+1=0 \\
p_{2}(t)=-t^{4}-t^{3}-t^{2}-2 t-1=0
\end{array}\right.
$$

The gcd of both polynomials is $H_{L}(t)=(t+1)$, and thus $\mathcal{F}_{\mathcal{P}}\left(P_{L}\right)=\{-1\}$ (i.e. $\left.\mathcal{P}(-1)=(0: 0: 1)=P_{L}\right)$. If we could apply Corollary 2.6, we would deduce that $P_{L}$ is a regular point since its multiplicity is 1 . However, this is not true. Indeed: let us consider the reparametrization $\mathcal{U}(t)=\mathcal{P}(1 / t)=$

$\left(t^{7}+2 t^{6}+3 t^{5}+4 t^{4}+3 t^{3}+2 t^{2}+t,-t^{7}-2 t^{6}-t^{5}-t^{4}-t^{3}, t^{7}+t^{6}+2 t^{5}+3 t^{4}+t^{3}+3 t^{2}+1\right)$.

Note that $\mathcal{U}(0)=(0: 0: 1)=P_{L}$. The fibre of $P_{L}$ via $\mathcal{U}(t), \mathcal{F}_{\mathcal{U}}\left(P_{L}\right)$, is given by the common roots of the equations

$$
\left\{\begin{array}{l}
q_{1}(t)=t^{7}+2 t^{6}+3 t^{5}+4 t^{4}+3 t^{3}+2 t^{2}+t=0 \\
q_{2}(t)=-t^{7}-2 t^{6}-t^{5}-t^{4}-t^{3}=0
\end{array}\right.
$$

The gcd of both polynomials is $H_{L}^{\mathcal{U}}(t)=t(t+1)$ and thus, the cardinality of the fibre, $\mathcal{F}_{\mathcal{U}}\left(P_{L}\right)$, is 2. Hence, $P_{L}$ is a double point of $\mathcal{C}$. The visible multiplicity is 1 , and the hidden multiplicity is 1.

In Figure 2, we plot the curve $\mathcal{C}$ in a neighborhood of the limit point $P_{L}$. More precisely, in Figure 2 (left), we plot $\mathcal{C}$ using $\mathcal{P}(t)$ with $-50 \leq t \leq 50$. In 

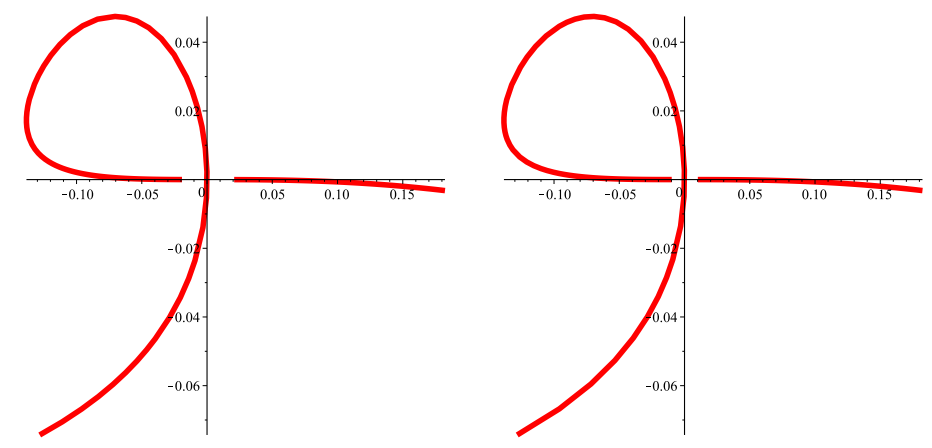

Figure 2: Curve $\mathcal{C}$ plotted from $\mathcal{P}(t)$ with $-50 \leq t \leq 50$ (left) and $-100 \leq t \leq 100$ (right)

Figure 2 (right), we plot $\mathcal{C}$ using $\mathcal{P}(t)$ with $-100 \leq t \leq 100$. Note that $P_{L}=$ $(0,0)$ is reached once by $\mathcal{P}(t)$ although it would be reached again in the limit, when $t$ tends to $\infty$. This second time (as the limit of the parametrization) does not have any fibre but it provides a second tangent (which increases the multiplicity of $P_{L}$ ) that is not detected from the parametrization $\mathcal{P}(t)$.

\section{The limit point and the $\mathrm{T}$-function}

In this section, we consider $\mathcal{C}$ a rational plane curve over an algebraically closed field of characteristic zero, $\mathbb{K}$, defined by the projective parametrization

$$
\mathcal{P}(t)=\left(p_{1}(t), p_{2}(t), p(t)\right) \in \mathbb{P}^{2}(\mathbb{K}(t)),
$$

where $\operatorname{gcd}\left(p_{1}, p_{2}, p\right)=1$. Let $d_{1}=\operatorname{deg}\left(p_{1}\right), d_{2}=\operatorname{deg}\left(p_{2}\right), d_{3}=\operatorname{deg}(p)$ and $d=\max \left\{d_{1}, d_{2}, d_{3}\right\}$. Then, $\operatorname{deg}(\mathcal{C})=d$.

In [2], a method for computing the singularities of $\mathcal{C}$ from $\mathcal{P}(t)$ is proposed. The method is based on the construction and factorization of a polynomial called the T-function, which may be defined in the following three ways:

$$
T(s)=\frac{R_{12}(s)}{p(s)^{\lambda_{12}-1}}=\frac{R_{13}(s)}{p_{1}(s)^{\lambda_{13}-1}}=\frac{R_{23}(s)}{p_{2}(s)^{\lambda_{23}-1}},
$$

where $\lambda_{i j}=\min \left\{\operatorname{deg}_{t}\left(G_{i}\right), \operatorname{deg}_{t}\left(G_{j}\right)\right\}, i, j \in\{1,2,3\}, i<j$, and

$$
R_{i j}(s)=\operatorname{Res}_{t}\left(\frac{G_{i}(s, t)}{t-s}, \frac{G_{j}(s, t)}{t-s}\right) .
$$


The $\mathrm{T}$-function provides essential information about the singularities of the given curve $\mathcal{C}$. More precisely, the factorization of the $\mathrm{T}$-function gives the fibre functions of the singularities of $\mathcal{C}$. We remark that from the fibre function of a point $P$, one may determine the multiplicity of $P$ as well as its fibre and the tangent lines at $P$. In [2], some important results concerning the $\mathrm{T}$-function are shown. In the following, we summarize the main theorem.

Theorem 4.1. (Theorem 3 in [2]) Let $\mathcal{C}$ be a rational algebraic plane curve defined by a parametrization $\mathcal{P}(t)$, with limit point $P_{L}$. Let $P_{1}, \ldots, P_{n}$ be the singular points of $\mathcal{C}$, with multiplicities $m_{1}, \ldots, m_{n}$ respectively. Let us assume that they are ordinary singularities and that $P_{i} \neq P_{L}$ for $i=1, \ldots, n$. Then, it holds that

$$
T(s)=\prod_{i=1}^{n} H_{P_{i}}(s)^{m_{i}-1}
$$

Theorem 4.1 holds under the assumption that the limit point, $P_{L}$, is regular. In Theorem 4.2 we eliminate this condition and generalize both results to the case that $P_{L}$ is a singularity. For this purpose, we state the following theorem whose proof will be presented in Section 5 .

Theorem 4.2. Let $\mathcal{C}$ be a rational algebraic plane curve of degree d defined by a proper parametrization $\mathcal{P}(t)$ with limit point $P_{L}$. Let $P_{1}, \ldots, P_{n}$ and $P_{L}$ be the singularities of $\mathcal{C}$ and suppose that all of them are ordinary. For each $P_{i}(i=1, \ldots, n)$, let $m_{i}$ be its multiplicity and $H_{P_{i}}$ its fibre function. In addition, let $m_{L}$ and $H_{L}$ be the multiplicity and the fibre function of $P_{L}$. Then,

$$
T(s)=\prod_{i=1}^{n} H_{P_{i}}(s)^{m_{i}-1} H_{L}(s)^{m_{L}-1} .
$$

Corollary 4.3. Let $m_{L}$ be the multiplicity of $P_{L}$ and $m_{H}$ the hidden multiplicity of $P_{L}$. It holds that

$$
\operatorname{deg}(T)=(d-1)(d-2)-m_{H}\left(m_{L}-1\right) .
$$

Proof: From Theorem 4.2, we deduce that

$$
\operatorname{deg}(T)=\sum_{i=1}^{n}\left(m_{i}-1\right) \operatorname{deg}\left(H_{P_{i}}\right)+\left(m_{L}-1\right) \operatorname{deg}\left(H_{L}\right)
$$


where $P_{1}, \ldots, P_{n}, P_{L}$ are the singularities of $\mathcal{C}$, and $m_{1}, \ldots, m_{n}, m_{L}$ their respective multiplicities. From Corollary 2.6, we get that $\operatorname{deg}\left(H_{P_{i}}\right)=m_{i}$. Furthermore, since $\operatorname{deg}\left(H_{L}\right)=m_{L}-m_{H}$ (see Definition 3.3), we get that

$$
\operatorname{deg}(T)=\sum_{i=1}^{n} m_{i}\left(m_{i}-1\right)+\left(m_{L}-m_{H}\right)\left(m_{L}-1\right) .
$$

Finally, the corollary follows using the genus formula (see Chapter 3 in [16]) which implies that

$$
\sum_{i=1}^{n} m_{i}\left(m_{i}-1\right)+m_{L}\left(m_{L}-1\right)=(d-1)(d-2) .
$$

Remark 4.4. 1. Theorem 4.2 shows that Theorem 4.1 holds regardless of wether $P_{L}$ is regular or not. If it is not regular, its fibre function appears as a factor of $T(s)$, raised to its multiplicity, as it happens for the other singularities. Besides, if it is singular, $m_{L}=1$ and the corresponding factor vanishes. Thus, we conclude that Theorem 4.1 may be stated without imposing any condition on the limit point.

2. By using Theorem 4.2, we get that:

(a) If for each factor $H_{P_{i}}(s)^{m_{i}-1}$ it holds that $\operatorname{deg}\left(H_{P_{i}}\right)=m_{i}, i=$ $1, \ldots, n$, then the limit point is regular.

(b) If there is a factor $H_{P_{i_{0}}}(s)^{m_{i_{0}}-1}$ such that $\operatorname{deg}\left(H_{P_{i_{0}}}\right)<m_{i_{0}}$, then $P_{i_{0}}$ is the limit point and $m_{H}=m_{i_{0}}-\operatorname{deg}\left(H_{P_{i_{0}}}\right)$.

3. Corollary 4.3 allows us to easily determine if the limit point is singular. In that case, we have that $\operatorname{deg}(T)<(d-1)(d-2)$; otherwise, if $\operatorname{deg}(T)=(d-1)(d-2)$, it means that $m_{L}=1$ and $P_{L}$ is regular.

Remark 4.5. In general, different conjugate roots of the T-function appear all together under a unique irreducible polynomial. These roots are associated to families of conjugated parametric points (see Definition 4 in [2]). In [12] (Theorem 16), it is shown that all the points in such a family have the same multiplicity and character. Let us assume that $T(s)$ includes a factor $m(s)^{k-1}$, where $m(s)$ is an irreducible polynomial of degree $l$. Then, $m(s)$ contains the fibre functions of $l / k$ singular points of multiplicity $k$ (see Theorem 5 in [2]). 
The following example shows how useful the above results are in order to study the singularities of a rational plane curve defined parametrically.

Example 4.6. In Example 3.6, we consider the rational plane curve $\mathcal{C}$ defined over $\mathbb{C}$ by the projective parametrization $\mathcal{P}(t)=$

$$
\left(t^{6}+2 t^{5}+3 t^{4}+3 t^{2}+4 t^{3}+2 t+1,-t^{4}-t^{3}-t^{2}-2 t-1, t^{7}+3 t^{5}+t^{4}+3 t^{3}+t+2 t^{2}+1\right) .
$$

We compute the T-function by applying (5), and we get that up to constants in $\mathbb{C}$,

$$
\begin{gathered}
T(s)=\left(s^{2}-s-1\right)\left(s^{3}+s+1\right)^{2} \\
\left(s^{8}+3 s^{7}+13 s^{6}+22 s^{5}+43 s^{4}+47 s^{3}+47 s^{2}+44 s+16\right)\left(s^{2}+1\right)^{6}(s+1) .
\end{gathered}
$$

In the following, we analyze each of the five factors of $T(s)$ :

- The first factor is $f_{1}(s)=s^{2}-s-1$. It has degree 2 and its power is 1 , which means that $f_{1}$ provides a double point. Indeed, $f_{1}$ has two real roots, $1 / 2+1 / 2 \sqrt{5}$ and $1 / 2-1 / 2 \sqrt{5}$. By substituting them into the parametrization we get the affine double point $P_{1}=(1:-1 / 5: 1)$.

- The second factor is $f_{2}(s)=\left(s^{3}+s+1\right)^{2}$. It has degree 3 and its power is 2, which means that $f_{2}$ provides a triple point. If we compute the three roots of $f_{2}$ and substitute them in the parametrization we get the infinity triple point $P_{2}=(1: 0: 0)$.

- The third factor is $f_{3}(s)=s^{8}+3 s^{7}+13 s^{6}+22 s^{5}+43 s^{4}+47 s^{3}+$ $47 s^{2}+44 s+16$. It is an irreducible polynomial of degree 8 and its power is 1. This implies that $f_{3}$ is associated to a set of double points which defines a family of conjugated parametric points (see Remark 4.5). More precisely, since the degree of the irreducible polynomial is 8 , we deduce that this family contains 4 double points.

- The fourth factor is $f_{4}(s)=\left(s^{2}+1\right)^{6}$. This factor may be difficult to interpret since one could think that the fibre function is $\left(s^{2}+1\right)$ and its power is 6. However, this is not correct. Actually, the fibre function is $\left(s^{2}+1\right)^{2}$ and its power is 3, which means that $f_{4}$ provides a point of multiplicity 4. In order to avoid mistakes, one has to compute one of the roots, namely $s=I$, the corresponding point of the curve, $P_{4}=\mathcal{P}(I)=(0: 1: 0)$, and the associate fibre function. From Remark 2.2, we get that $H_{P_{4}}(s)=\left(s^{2}+1\right)^{2}$. 
- The last factor is $f_{5}(s)=s+1$, and it provides the point $P_{5}=\mathcal{P}(-1)=$ $(0: 0: 1)$. The fibre function of $P_{5}$ is $H_{P_{5}}(s)=s+1$. Observe that $H_{P_{5}}$ has degree 1 and its power is 1 . This situation is described in statement 2 (b) of Remark 4.4. More precisely, we have that $m_{5}=$ $2>\operatorname{deg}\left(H_{P_{5}}\right)=1$, which implies that $P_{5}$ is the limit point. Its total multiplicity is $m_{L}=2$ and its hidden multiplicity is $m_{H}=1$ (see Definition 3.3).

We observe that since $\mathcal{C}$ is rational and the singularities are ordinary, it holds that

$$
g=(d-1)(d-2) / 2-\sum_{i=1}^{k} m_{i}\left(m_{i}-1\right) / 2, \quad \text { and } g=0
$$

where $g$ is the genus of $\mathcal{C}, d$ is the degree of the given curve, and $P_{i}, i=$ $1, \ldots, k$ are the ordinary singular points of multiplicity, $m_{i}, i=1, \ldots, k$, respectively. Observe that a point $P_{i}$ of multiplicity $m_{i}$ degenerates to $m_{i}\left(m_{i}-\right.$ 1)/2 double points. In this particular example, one may check that this formulae holds since the point of multiplicity 3 degenerates to 3 double points, the point of multiplicity 4 degenerates to 6 double points, and thus, since $d=7$ and $g=0$, it holds that $3+6+4+2=15=6 \cdot 5 / 2$.

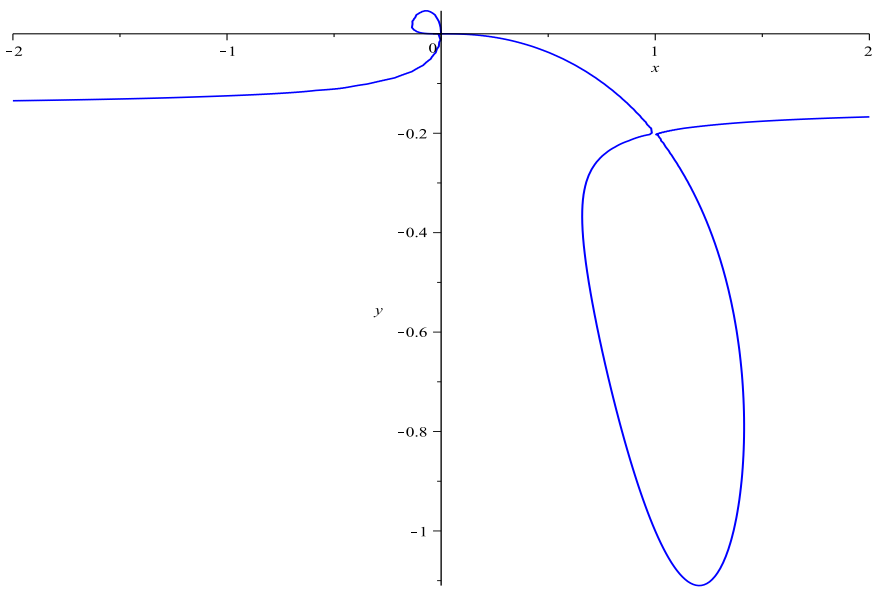

Figure 3: The curve $\mathcal{C}$ has two real affine double points at $(1:-1 / 5: 1)$ and $(0: 0: 1)$

In Figure 3, we plot the curve $\mathcal{C}$. One may see the two real affine double points $P_{1}$ and $P_{5}$. 
As we state in the Introduction, using the ideas presented in Section 4 in [2], one may easily generalize the results presented in this paper to the case of a given rational space curve in any dimension. In particular, one may construct a polynomial, $T_{E}(s)$, that plays the same role as the T-function, $T(s)$, introduced for the case of rational plane curves. Thus, a similar result to the stated in Theorem 4.2 can be obtained for rational space curves.

\section{Complexity of the computation}

As we stated in the introduction, one of the main topics in the study of algebraic varieties, in particular curves and surfaces, from the parametric point of view, is the analysis of singularities. Here, rational parametrizations provide interesting approaches from the computational point of view.

For instance, for the case of parametric plane curves, some interesting results are provided in [4], where the singular points are computed using the implicitization matrix derived from the $\mu$-basis of the curve. In addition, a conjecture is presented which concerns the computation of the parameter values corresponding to all the singularities, from the Smith normal forms of certain Bézout resultant matrices derived from $\mu$-bases. In [19], a natural one to one correspondence is derived between the singular points of a rational planar curve and the axial moving lines that follow that curves. This correspondence is applied to compute and analyze the singular points of low degree rational planar curves, by using $\mu$-bases. The $\mu$-basis approach is also used in [3], where it is given a complete factorization of the invariant factors of certain resultant matrices, built from birational parameterizations of rational plane curves, in terms of the singular points of the curve and their multiplicity graph. This also allows to prove the validity of some conjectures introduced in [4]. A new technique for detecting singularities is introduced in [18]. The idea is to compute a $\mu$-basis for the parametrization and to generate, from this $\mu$-basis, three planar algebraic curves of different bidegrees, whose intersection points correspond to the parameters of the singularities. In order to find these intersection points, a new sparse resultant matrix for these three bivariate polynomials is constructed. Afterwards, authors compute the parameter values corresponding to the singularities by applying Gaussian elimination to the resultant matrix.

All these works are based on the use of $\mu$-basis. Besides, there is a second line of work, based on the use of univariate resultants. For instance, in [12], 
a method for detecting and analyzing the singularities of a rational curve (including the non-ordinary ones) by computing a univariate resultant, is provided. This approach is based on the ideas introduced in Subsection 4.3 in [16], and it generalizes some previous results presented in [1], [5], [10] and [20]. In [2], some important results concerning the analysis of the singularities are also presented. These results are based on the paper [12] and hence, the main technique used is the computation of a univariate resultant. We recall that in this paper, we generalize the results in [2] concerning the T-function, $T(s)$, which is defined by means of a univariate resultant.

On the other side, the study of singularities in parametric space curves has been addressed in [2], [14], [18] and [19]. In this case, also the most important methods used have to do with the computation of $\mu$-bases and univariate resultants. For the case of surfaces, some works have also been published (see e.g. [13]).

The results presented in this paper are based on the computation of only one univariate resultant and the previous results summarized above depend on the computation of $\mu$-basis and afterwards, in general, an additional resultant has to be computed. Thus, given a rational plane curve $\mathcal{C}$, of degree $d$, defined by the projective parametrization $\mathcal{P}(t)=\left(p_{1}(t), p_{2}(t), p(t)\right)$ the complexity of computing the T-function is $\mathcal{O}\left(d^{3}\right)$. The methods based on the computation of $\mu$-basis need to compute the $\mu$-basis which complexity is $\mathcal{O}\left(d^{3}\right)$, and the resultant of the $\mu$-basis which complexity is $\mathcal{O}\left(d^{2}\right)$. We note that the degrees of the $\mu$-basis, $q_{1}(t)=\left(q_{11}, q_{12}, q_{13}\right)$ and $q_{2}(t)=\left(q_{21}, q_{22}, q_{23}\right)$, are $d_{1}$ and $d_{2}$ with $d_{1}+d_{2}=d$ and thus, the size of resultant of $\mu$-basis is smaller than that the resultant needed to compute the T-function. Observe that a $\mu$-basis is a basis of syzygy module with lowest degrees and the polynomials $G_{i}(t, s), i=1,2$ introduced in this paper (see (1)) are defined from $\left(p, 0,-p_{1}\right)$ and $\left(0, p,-p_{2}\right)$ which are called trivial syzygy of $\mathcal{P}(t)$ and they can be generated by a $\mu$-basis.

Therefore, one may conclude that the time complexity of the different existing methods is $\mathcal{O}\left(d^{3}\right)$, where $d$ is the degree of the input curve. 


\section{Proof of Theorem 4.2 in Section 4}

This section is devoted to prove Theorem 4.2 stated in Section 4. For this purpose, throughout this section, we use the reparametrization

$$
\mathcal{Q}(t):=\mathcal{P}\left(\frac{\theta t}{t-1}\right)
$$

introduced in Remark 3.4. Observe that

$$
\mathcal{Q}(t)=\left(p_{1}\left(\frac{\theta t}{t-1}\right)(t-1)^{d}, p_{2}\left(\frac{\theta t}{t-1}\right)(t-1)^{d}, p\left(\frac{\theta t}{t-1}\right)(t-1)^{d}\right)
$$

and then, we may write $\mathcal{Q}(t)=\left(q_{1}(t), q_{2}(t), q(t)\right)$, where

$$
\left\{\begin{array}{c}
q_{1}(t)=a_{0}(t-1)^{d}+a_{1} \theta t(t-1)^{d-1}+\cdots+a_{d} \theta^{d} t^{d} \\
q_{2}(t)=b_{0}(t-1)^{d}+b_{1} \theta t(t-1)^{d-1}+\cdots+b_{d} \theta^{d} t^{d} \\
q(t)=c_{0}(t-1)^{d}+c_{1} \theta t(t-1)^{d-1}+\cdots+c_{d} \theta^{d} t^{d}
\end{array}\right.
$$

Note that, with this parametrization, $P_{L}$ is not the limit point but a conventional point which can be obtained as $P_{L}=\mathcal{Q}(1)$. The new limit point is

$$
Q_{L}=\lim _{t \rightarrow \infty} \mathcal{Q}(t) / t^{d}=\mathcal{P}(\theta),
$$

and we can choose $\theta \in \mathbb{K} \backslash\{0\}$ so that $Q_{L}$ is not a singularity. In addition, if $p(\theta) \neq 0$, we get that $Q_{L}$ is an affine regular point. Note that every point of the curve reached via $\mathcal{P}(t)$, but $Q_{L}$, is also reached via $\mathcal{Q}(t)$. Indeed, for any $s_{0} \neq \theta$ it holds that $\mathcal{Q}\left(\frac{s_{0}}{s_{0}-\theta}\right)=\mathcal{P}\left(s_{0}\right)$. The only exception arises when $s_{0}=\theta$, since $\mathcal{P}(\theta)=Q_{L}$.

Taking into account this last statement, we have that for each $s_{i} \in \mathcal{F}_{\mathcal{P}}\left(P_{L}\right)$ there exists $t_{i}=s_{i} /\left(s_{i}-\theta\right) \in \mathcal{F}_{\mathcal{Q}}\left(P_{L}\right)$ (note that $s_{i} \neq \theta$ since $\theta \notin \mathcal{F}_{\mathcal{P}}\left(P_{L}\right)$; we recall that $\left.\mathcal{P}(\theta)=Q_{L} \neq P_{L}\right)$. However, there is a value in $\mathcal{F}_{\mathcal{Q}}\left(P_{L}\right)$ which does not have a correspondence in $\mathcal{F}_{\mathcal{P}}\left(P_{L}\right)$; namely $t=1$. Note that this value belongs to $\mathcal{F}_{\mathcal{Q}}\left(P_{L}\right)$, since $\mathcal{Q}(1)=P_{L}$, but there is no $s_{i} \in \mathbb{K}$ such that $\frac{s_{i}}{s_{i}-\theta}=1$ (note that $\theta \in \mathbb{K} \backslash\{0\}$ ). As a consequence, the fibre function of $P_{L}$ under the parametrization $\mathcal{Q}(t)$ is

$$
\widetilde{H}_{L}(t)=(t-1)^{r} \prod_{i=1}^{n}\left(t-\frac{s_{i}}{s_{i}-\theta}\right)^{k_{i}}
$$


where $k_{1}+\cdots+k_{n}$ is the visible multiplicity and $r$ is the hidden multiplicity (see the analogy with (4)). Hence, we observe that $\operatorname{deg}\left(\widetilde{H}_{L}\right)=k_{1}+\cdots+k_{n}+$ $r=\operatorname{deg}\left(H_{L}\right)+m_{H}=m_{L}$ (see Definition 3.3).

Summarizing, we have a new parametrization of $\mathcal{C}$ such that the limit point, $Q_{L}$, is regular; this allows us to apply the results obtained in [2]. For this purpose, let $\widetilde{G}_{1}(s, t), \widetilde{G}_{2}(s, t)$ and $\widetilde{G}_{3}(s, t)$ be the equivalent polynomials to $G_{1}(s, t), G_{2}(s, t)$ and $G_{3}(s, t)$ (see $(1)$ ) computed from the new parametrization $\mathcal{Q}(t)$. That is,

$$
\left\{\begin{array}{l}
\widetilde{G}_{1}(s, t)=q_{1}(s) q(t)-q(s) q_{1}(t) \\
\widetilde{G}_{2}(s, t)=q_{2}(s) q(t)-q(s) q_{2}(t) \\
\widetilde{G}_{3}(s, t)=q_{1}(s) q_{2}(t)-q_{2}(s) q_{1}(t) .
\end{array}\right.
$$

In addition, let $\widetilde{\delta}_{i}=\operatorname{deg}_{t}\left(\widetilde{G}_{i}\right), \widetilde{\lambda}_{i j}=\min \left\{\widetilde{\delta}_{i}, \widetilde{\delta}_{j}\right\}, i, j=1,2,3$, and

$$
\widetilde{R}_{i j}(s)=\operatorname{Res}_{t}\left(\frac{\widetilde{G}_{i}(s, t)}{t-s}, \frac{\widetilde{G}_{j}(s, t)}{t-s}\right), \quad \text { for } i, j=1,2,3, i<j .
$$

The $\mathrm{T}$-function obtained from $\mathcal{Q}$ is

$$
\widetilde{T}=\widetilde{R}_{12} / q^{\widetilde{\lambda}_{12}-1}=\widetilde{R}_{13} / q_{1}^{\widetilde{\lambda}_{13}-1}=\widetilde{R}_{23} / q_{2}^{\widetilde{\lambda}_{23}-1} .
$$

Now, Theorem 4.1 states that

$$
\widetilde{T}(s)=\prod_{i=1}^{n} \widetilde{H}_{P_{i}}(s)^{m_{i}-1} \widetilde{H}_{L}(s)^{m_{L}-1}
$$

and $\operatorname{deg}(\widetilde{T})=(d-1)(d-2)\left(Q_{L}\right.$ is regular and we apply Corollary 3 in $\left.[2]\right)$.

We will use both statements later but, before, let us introduce the following technical lemma, which describes the relation between $R_{12}$ and $\widetilde{R}_{12}$ under the assumption that $\operatorname{deg}_{t}\left(G_{1}\right)=\operatorname{deg}_{t}\left(G_{2}\right)$.

Lemma 5.1. Let $\operatorname{deg}_{t}\left(G_{1}\right)=\operatorname{deg}_{t}\left(G_{2}\right)$. Then, it holds that

$$
\widetilde{R}_{12}(s)=(s-1)^{2(d-1)^{2}} R_{12}\left(\frac{\theta s}{s-1}\right) .
$$


Proof: We prove the lemma by considering two steps. In the first one, we obtain the form of $R_{12}\left(\frac{\theta s}{s-1}\right)$. In the second one, we compute $\widetilde{R}_{12}(s)$ and we compare it with $R_{12}\left(\frac{\theta s}{s-1}\right)$.

\section{Step 1}

First, we recall that

$$
R_{12}(s)=\operatorname{Res}_{t}\left(\frac{G_{1}(s, t)}{t-s}, \frac{G_{2}(s, t)}{t-s}\right),
$$

where $G_{1}(s, t)=p_{1}(s) p(t)-p(s) p_{1}(t)$ and $G_{2}(s, t)=p_{2}(s) p(t)-p(s) p_{2}(t)$. In addition, since $\delta_{1}=\delta_{2}$, where $\delta_{1}:=\operatorname{deg}_{t}\left(G_{1}\right)$ and $\delta_{2}:=\operatorname{deg}_{t}\left(G_{2}\right)$, and taking into account that $\delta_{1}=\max \left\{d_{1}, d_{3}\right\}$ and $\delta_{2}=\max \left\{d_{2}, d_{3}\right\}$ (see Remark 1 in [2]), we get that $\delta_{1}=\delta_{2}=\max \left\{d_{1}, d_{2}, d_{3}\right\}=d$.

In the following, we denote $G_{1}^{*}:=G_{1} /(t-s)$ and $G_{2}^{*}:=G_{2} /(t-s)$, and thus $R_{12}(s)=\operatorname{Res}_{t}\left(G_{1}^{*}, G_{2}^{*}\right)$, where $\operatorname{deg}_{t}\left(G_{1}^{*}\right)=\operatorname{deg}_{t}\left(G_{2}^{*}\right)=d-1$ (note that $\left.\operatorname{deg}_{s}\left(G_{1}^{*}\right)=\operatorname{deg}_{s}\left(G_{2}^{*}\right)=d-1\right)$. If we see $G_{1}^{*}$ and $G_{2}^{*}$ as polynomials in the variable $t$, that is, $G_{1}^{*}, G_{2}^{*} \in(\mathbb{K}[s])[t]$, we may write

$$
G_{1}^{*}(s, t)=\mathrm{lc}_{t}\left(G_{1}^{*}\right) \prod_{i=1}^{d-1}\left(t-\alpha_{i}(s)\right) \text { and } G_{2}^{*}(s, t)=\mathrm{lc}_{t}\left(G_{2}^{*}\right) \prod_{j=1}^{d-1}\left(t-\beta_{j}(s)\right),
$$

where $\mathrm{lc}_{t}\left(G_{1}^{*}\right)$ and $\mathrm{lc}_{t}\left(G_{2}^{*}\right)$ are their respective leader coefficients in $\mathbb{K}[s]$ and $\alpha_{1}(s), \ldots, \alpha_{d-1}(s)$ and $\beta_{1}(s), \ldots, \beta_{d-1}(s)$ their $d-1$ roots, respectively (that is, $G_{1}^{*}\left(s, \alpha_{i}(s)\right)=G_{2}^{*}\left(s, \beta_{j}(s)\right)=0$ for $\left.i, j=1, \ldots, d-1\right)$. Now, taking into account the properties of the resultant, we have that

$$
R_{12}(s)=\mathrm{lc}_{t}\left(G_{1}^{*}\right)^{d-1} \mathrm{lc}_{t}\left(G_{2}^{*}\right)^{d-1} \prod_{i=1}^{d-1} \prod_{j=1}^{d-1}\left(\alpha_{i}(s)-\beta_{j}(s)\right) .
$$

By substituting $t=\theta$ in (13), we obtain

$$
G_{1}^{*}(s, \theta)=\mathrm{lc}_{t}\left(G_{1}^{*}\right) \prod_{i=1}^{d-1}\left(\theta-\alpha_{i}(s)\right) \text { and } G_{2}^{*}(s, \theta)=\mathrm{lc}_{t}\left(G_{2}^{*}\right) \prod_{j=1}^{d-1}\left(\theta-\beta_{j}(s)\right)
$$

so, up to constants in $\mathbb{K} \backslash\{0\}$,

$$
\operatorname{lc}_{t}\left(G_{1}^{*}\right)=\frac{G_{1}^{*}(s, \theta)}{\prod_{i=1}^{d-1}\left(\alpha_{i}(s)-\theta\right)} \quad \text { and } \quad \operatorname{lc}_{t}\left(G_{2}^{*}\right)=\frac{G_{2}^{*}(s, \theta)}{\prod_{j=1}^{d-1}\left(\beta_{j}(s)-\theta\right)} .
$$


In the following, we denote $\Phi_{1}(s):=\prod_{i=1}^{d-1}\left(\alpha_{i}(s)-\theta\right)$ and $\Phi_{2}(s):=$ $\prod_{j=1}^{d-1}\left(\beta_{j}(s)-\theta\right)$. Thus, taking into account that $G_{i}^{*}(s, t):=G_{i}(s, t) /(t-s)$, we get that

$$
\mathrm{lc}_{t}\left(G_{1}^{*}\right)=\frac{G_{1}(s, \theta)}{(s-\theta) \Phi_{1}(s)} \quad \text { and } \quad \operatorname{lc}_{t}\left(G_{2}^{*}\right)=\frac{G_{2}(s, \theta)}{(s-\theta) \Phi_{2}(s)} .
$$

By substituting both expressions in (14), we obtain that

$$
R_{12}(s)=\left(\frac{G_{1}(s, \theta)}{(s-\theta) \Phi_{1}(s)}\right)^{d-1}\left(\frac{G_{2}(s, \theta)}{(s-\theta) \Phi_{2}(s)}\right)^{d-1} \prod_{i=1}^{d-1} \prod_{j=1}^{d-1}\left(\alpha_{i}(s)-\beta_{j}(s)\right)
$$

and therefore,

$$
\begin{gathered}
R_{12}\left(\frac{\theta s}{s-1}\right)=\left(\frac{G_{1}\left(\frac{\theta s}{s-1}, \theta\right)}{\left(\frac{\theta s}{s-1}-\theta\right) \Phi_{1}\left(\frac{\theta s}{s-1}\right)}\right)^{d-1} \\
\left(\frac{G_{2}\left(\frac{\theta s}{s-1}, \theta\right)}{\left(\frac{\theta s}{s-1}-\theta\right) \Phi_{2}\left(\frac{\theta s}{s-1}\right)}\right)^{d-1} \prod_{i=1}^{d-1} \prod_{j=1}^{d-1}\left(\alpha_{i}\left(\frac{\theta s}{s-1}\right)-\beta_{j}\left(\frac{\theta s}{s-1}\right)\right) \\
=\left(\frac{G_{1}\left(\frac{\theta s}{s-1}, \theta\right)}{\Phi_{1}\left(\frac{\theta s}{s-1}\right)}\right)^{d-1}\left(\frac{G_{2}\left(\frac{\theta s}{s-1}, \theta\right)}{\Phi_{2}\left(\frac{\theta s}{s-1}\right)}\right)^{d-1}\left(\frac{s-1}{\theta}\right)^{2(d-1)} \\
\prod_{i=1}^{d-1} \prod_{j=1}^{d-1}\left(\alpha_{i}\left(\frac{\theta s}{s-1}\right)-\beta_{j}\left(\frac{\theta s}{s-1}\right)\right) .
\end{gathered}
$$

Step 2

First, we observe that the coefficient of the term $t^{d}$ in $q(t)$ is $p(\theta)$. Thus, $\operatorname{deg}(q)=d$ (note that $\theta$ is such that $p(\theta) \neq 0$ ) and hence, $\operatorname{deg}_{t}\left(\widetilde{G}_{1}\right)=d$. Indeed: we have that $\widetilde{G}_{1}(s, t)=q_{1}(s) q(t)-q(s) q_{1}(t)$ (see $(10)$ ), so it follows that $\operatorname{deg}_{t}\left(\widetilde{G}_{1}\right)=d$ if $\operatorname{deg}\left(q_{1}\right)<d$. On the other hand, if $\operatorname{deg}\left(q_{1}\right)=d$, it could happen that $\operatorname{deg}_{t}\left(\widetilde{G}_{1}\right)<d$ if $q_{1}(s) q(\theta)-q(s) q_{1}(\theta)=0$ but this would imply that $q_{1}(s) / q(s)$ is a constant and, thus, $\mathcal{C}$ is a line, which is impossible by assumption. Reasoning similarly, we deduce that $\operatorname{deg}_{t}\left(\widetilde{G}_{2}\right)=d$.

Let $\widetilde{G}_{1}^{*}:=\widetilde{G}_{1} /(t-s)$ and $\widetilde{G}_{2}^{*}:=\widetilde{G}_{2} /(t-s)$. Then, $\widetilde{R}_{12}(s)=\operatorname{Res}_{t}\left(\widetilde{G}_{1}^{*}, \widetilde{G}_{2}^{*}\right)$. Similarly as before, we have that, $\widetilde{G}_{1}^{*}$ and $\widetilde{G}_{2}^{*}$ are polynomials in the variables 
$s$ and $t$, with degree $d-1$ in both variables. Moreover, reasoning as in Step 1 , we have that

$$
\widetilde{G}_{1}^{*}(s, t)=\mathrm{lc}_{t}\left(\widetilde{G}_{1}^{*}\right) \prod_{i=1}^{d-1}\left(t-\widetilde{\alpha}_{i}(s)\right), \quad \widetilde{G}_{2}^{*}(s, t)=\mathrm{lc}_{t}\left(\widetilde{G}_{2}^{*}\right) \prod_{j=1}^{d-1}\left(t-\widetilde{\beta}_{j}(s)\right),
$$

and

$$
\widetilde{R}_{12}(s)=\mathrm{lc}_{t}\left(\widetilde{G}_{1}^{*}\right)^{d-1} \mathrm{lc}_{t}\left(\widetilde{G}_{2}^{*}\right)^{d-1} \prod_{i=1}^{d-1} \prod_{j=1}^{d-1}\left(\widetilde{\alpha}_{i}(s)-\widetilde{\beta}_{j}(s)\right) .
$$

Note also that $\mathrm{lc}_{t}\left(\widetilde{G}_{1}^{*}\right)=\mathrm{lc}_{t}\left(\widetilde{G}_{1}\right)=$

$q_{1}(s)\left(c_{0}+c_{1} \theta+\cdots+c_{d} \theta^{d}\right)-q(s)\left(a_{0}+a_{1} \theta+\cdots+a_{d} \theta^{d}\right)=q_{1}(s) p(\theta)-q(s) p_{1}(\theta)$

which can be written as

$p_{1}(\theta s /(s-1))(s-1)^{d} p(\theta)-p(\theta s /(s-1))(s-1)^{d} p_{1}(\theta)=G_{1}(\theta s /(s-1), \theta)(s-1)^{d}$.

Reasoning similarly with $\operatorname{lc}_{t}\left(\widetilde{G}_{2}^{*}\right)$, we conclude that

$$
\mathrm{lc}_{t}\left(\widetilde{G}_{1}^{*}\right)=G_{1}\left(\frac{\theta s}{s-1}, \theta\right)(s-1)^{d} \text { and } \operatorname{lc}_{t}\left(\widetilde{G}_{2}^{*}\right)=G_{2}\left(\frac{\theta s}{s-1}, \theta\right)(s-1)^{d} .
$$

Now, we focus on the roots $\widetilde{\alpha}_{1}(s), \ldots, \widetilde{\alpha}_{d-1}(s)$. On the one side, all of them verify that $\widetilde{G}_{1}^{*}\left(s, \widetilde{\alpha}_{i}(s)\right)=0$. On the other side, note that

$$
\begin{gathered}
\widetilde{G}_{1}(s, t)=q_{1}(s) q(t)-q(s) q_{1}(t)= \\
=p_{1}\left(\frac{\theta s}{s-1}\right)(s-1)^{d} p\left(\frac{\theta t}{t-1}\right)(t-1)^{d}-p\left(\frac{\theta s}{s-1}\right)(s-1)^{d} p_{1}\left(\frac{\theta t}{t-1}\right)(t-1)^{d} \\
=(s-1)^{d}(t-1)^{d} G_{1}\left(\frac{\theta s}{s-1}, \frac{\theta t}{t-1}\right) .
\end{gathered}
$$

Thus, $\widetilde{G}_{1}^{*}\left(s, \widetilde{\alpha}_{i}(s)\right)=0$, which is equivalent to

$$
G_{1}\left(\frac{\theta s}{s-1}, \frac{\theta \widetilde{\alpha}_{i}(s)}{\widetilde{\alpha}_{i}(s)-1}\right)=0
$$

implies that

$$
\alpha_{i}\left(\frac{\theta s}{s-1}\right)=\frac{\theta \widetilde{\alpha}_{i}(s)}{\widetilde{\alpha}_{i}(s)-1}
$$


and thus

$$
\widetilde{\alpha}_{i}(s)=\frac{\alpha_{i}\left(\frac{\theta s}{s-1}\right)}{\alpha_{i}\left(\frac{\theta s}{s-1}\right)-\theta} .
$$

Reasoning similarly with $\widetilde{\beta}_{1}(s), \ldots, \widetilde{\beta}_{d-1}(s)$, we deduce that

$$
\widetilde{\alpha}_{i}(s)=\frac{\alpha_{i}\left(\frac{\theta s}{s-1}\right)}{\alpha_{i}\left(\frac{\theta s}{s-1}\right)-\theta} \quad \text { and } \quad \widetilde{\beta}_{j}(s)=\frac{\beta_{j}\left(\frac{\theta s}{s-1}\right)}{\beta_{j}\left(\frac{\theta s}{s-1}\right)-\theta}
$$

for each $i, j=1, \ldots, d-1$.

Now, by substituting (22) and (23) on (21), we get

$$
\begin{aligned}
& \widetilde{R}_{12}(s)=\left(G_{1}\left(\frac{\theta s}{s-1}, \theta\right)(s-1)^{d}\right)^{d-1}\left(G_{2}\left(\frac{\theta s}{s-1}, \theta\right)(s-1)^{d}\right)^{d-1} \\
& \prod_{i=1}^{d-1} \prod_{j=1}^{d-1}\left(\frac{\alpha_{i}\left(\frac{\theta s}{s-1}\right)}{\alpha_{i}\left(\frac{\theta s}{s-1}\right)-\theta}-\frac{\beta_{j}\left(\frac{\theta s}{s-1}\right)}{\beta_{j}\left(\frac{\theta s}{s-1}\right)-\theta}\right)= \\
&= G_{1}\left(\frac{\theta s}{s-1}, \theta\right)^{d-1} G_{2}\left(\frac{\theta s}{s-1}, \theta\right)^{d-1}(s-1)^{2 d(d-1)} \\
& \prod_{i=1}^{d-1} \prod_{j=1}^{d-1}\left(\frac{\alpha_{i}\left(\frac{\theta s}{s-1}\right)\left(\beta_{j}\left(\frac{\theta s}{s-1}\right)-\theta\right)-\beta_{j}\left(\frac{\theta s}{s-1}\right)\left(\alpha_{i}\left(\frac{\theta s}{s-1}\right)-\theta\right)}{\left(\alpha_{i}\left(\frac{\theta s}{s-1}\right)-\theta\right)\left(\beta_{j}\left(\frac{\theta s}{s-1}\right)-\theta\right)}\right)= \\
&= G_{1}\left(\frac{\theta s}{s-1}, \theta\right)^{d-1} G_{2}\left(\frac{\theta s}{s-1}, \theta\right)^{d-1}(s-1)^{2 d(d-1)} \\
& \frac{\prod_{i=1}^{d-1} \prod_{j=1}^{d-1}\left(\alpha_{i}\left(\frac{\theta s}{s-1}\right)-\beta_{j}\left(\frac{\theta s}{s-1}\right)\right) \theta}{\prod_{i=1}^{d-1}\left(\alpha_{i}\left(\frac{\theta s}{s-1}\right)-\theta\right)^{d-1} \prod_{j=1}^{d-1}\left(\beta_{j}\left(\frac{\theta s}{s-1}\right)-\theta\right)^{d-1}=} \\
&=\left(\frac{G_{1}\left(\frac{\theta s}{s-1}, \theta\right)}{\Phi_{1}\left(\frac{\theta s}{s-1}\right)}\right)^{d-1}\left(\frac{G_{2}\left(\frac{\theta s}{s-1}, \theta\right)}{\Phi_{2}\left(\frac{\theta s}{s-1}\right)}\right)^{d-1}(s-1)^{2 d(d-1)} \theta^{(d-1)^{2}} \\
& \prod_{i=1}^{d-1} \prod_{j=1}^{d-1}\left(\alpha_{i}\left(\frac{\theta s}{s-1}\right)-\beta_{j}\left(\frac{\theta s}{s-1}\right)\right)
\end{aligned}
$$


Finally, by comparing this expression with (19), we observe that, up to constants,

$$
\frac{\widetilde{R}_{12}(s)}{(s-1)^{2 d(d-1)}}=\frac{R_{12}(\theta s /(s-1))}{(s-1)^{2(d-1)}}
$$

which proves the lemma.

Remark 5.2. Reasoning as in the proof of Lemma 5.1, one shows that

1. If $p_{1}(\theta) \neq 0$, then $\widetilde{R}_{13}(s)=(s-1)^{2(d-1)^{2}} R_{13}\left(\frac{\theta s}{s-1}\right)$.

2. If $p_{2}(\theta) \neq 0$, then $\widetilde{R}_{23}(s)=(s-1)^{2(d-1)^{2}} R_{23}\left(\frac{\theta s}{s-1}\right)$.

Now, we are ready to deal with the main item of the section. More precisely, we prove Theorem 4.2 (see Section 4), where Theorem 4.1 is generalized to the case that the limit point, $P_{L}$, is a singularity of the curve. In order to show this result, Lemma 5.1 and Remark 5.2 will be required.

\section{Proof of Theorem 4.2}

We consider two steps in the proof of the theorem. First, we assume that $\delta_{1}=\delta_{2}$, which allows us to use Lema 5.1 and second, we eliminate this requirement and we prove that the result holds anyway.

\section{Step 1}

According to Lema 2 in [2], for each singularity $P_{i}, i=1, \ldots, n$ it holds that $T(s)=H_{P_{i}}(s)^{m_{i}-1} T_{i}^{*}(s)$, where $T_{i}^{*}$ is a polynomial such that $\operatorname{gcd}\left(H_{P_{i}}, T_{i}^{*}\right)=$ 1. Note that $\operatorname{gcd}\left(H_{P_{i}}, H_{P_{j}}\right)=1$ for $i \neq j$ (otherwise, one single value of the parameter would generate two different points of the curve). Hence, we have that

$$
T(s)=\prod_{i=1}^{n} H_{P_{i}}(s)^{m_{i}-1} V(s),
$$

where $V(s)$ is a polynomial such that $\operatorname{gcd}\left(H_{P_{i}}, V\right)=1$, for $i=1, \ldots, n$.

Now, we focus on the polynomial $V$. We observe that if $V\left(s_{0}\right)=0$ then $T\left(s_{0}\right)=0$ which implies that $R_{12}\left(s_{0}\right)=R_{13}\left(s_{0}\right)=R_{23}\left(s_{0}\right)=0$ (see (5)). Moreover, we note that $d=\max \left\{d_{1}, d_{2}, d_{3}\right\}$, so it can not happen that $a_{d}=b_{d}=c_{d}=0$. In the following we assume w.l.o.g., that $a_{d} \neq 0$ and we consider $R_{13}$ (if $a_{d}=0$ and $b_{d} \neq 0$, we would consider $R_{23}$ and if $a_{d}=b_{d}=0$ 
and $c_{d} \neq 0$ we would use $R_{12}$ ). Then, let $a_{d} \neq 0$. We have that if $V\left(s_{0}\right)=0$, then

$$
R_{13}\left(s_{0}\right)=\operatorname{Res}_{t}\left(G_{1}^{*}(s, t), G_{3}^{*}(s, t)\right)\left(s_{0}\right)=0,
$$

and thus, one of the following statements hold:

1. The polynomials $G_{1}^{*}\left(s_{0}, t\right)$ and $G_{3}^{*}\left(s_{0}, t\right)$ have a common root, say $t=$ $s_{1} \neq s_{0}$, which implies that the point $P=\mathcal{P}\left(s_{0}\right)=\mathcal{P}\left(s_{1}\right)$ is a singularity. However, it can not be $P=P_{i}$ for $i=1, \ldots, n$ since it would imply that $\operatorname{gcd}\left(H_{P_{i}}, V\right) \neq 1$ (both polynomials would have $\left(t-s_{0}\right)$ as a common factor). Thus, we have that $\mathcal{P}\left(s_{0}\right)=P_{L}$ and $H_{L}\left(s_{0}\right)=0$.

2. It holds that $\operatorname{gcd}\left(\operatorname{lc}_{t}\left(G_{1}^{*}\right), \operatorname{lc}_{t}\left(G_{3}^{*}\right)\right)\left(s_{0}\right)=0$. Note that

$$
\begin{gathered}
\operatorname{lc}_{t}\left(G_{1}^{*}\right)=\operatorname{lc}_{t}\left(G_{1}\right)=p_{1}(s) c_{d}-p(s) a_{d}=\phi_{1}^{L}(s) \text { and } \\
\operatorname{lc}_{t}\left(G_{3}^{*}\right)=\operatorname{lc}_{t}\left(G_{3}\right)=p_{1}(s) b_{d}-p_{2}(s) a_{d}=\phi_{3}^{L}(s)
\end{gathered}
$$

and then, $\operatorname{gcd}\left(\mathrm{lc}_{t}\left(G_{1}^{*}\right), \mathrm{lc}_{t}\left(G_{3}^{*}\right)\right)=\operatorname{gcd}\left(\phi_{1}^{L}(s), \phi_{3}^{L}(s)\right)$. In addition, since we are assuming that $a_{d} \neq 0$, we may write

$$
\phi_{2}^{L}(s)=\frac{b_{d}}{a_{d}} \phi_{1}^{L}(s)-\frac{c_{d}}{a_{d}} \phi_{3}^{L}(s),
$$

and, hence, $\operatorname{gcd}\left(\phi_{1}^{L}(s), \phi_{3}^{L}(s)\right)=\operatorname{gcd}\left(\phi_{1}^{L}(s), \phi_{2}^{L}(s), \phi_{3}^{L}(s)\right)=H_{L}(s)$. Finally we conclude that

$$
\operatorname{gcd}\left(\mathrm{lc}_{t}\left(G_{1}^{*}\right), \mathrm{lc}_{t}\left(G_{3}^{*}\right)\right)=H_{L}(s) .
$$

In both cases, $V\left(s_{0}\right)=0$ implies that $H_{L}\left(s_{0}\right)=0$. Now, let us check that the reciprocal holds, that is, we assume that $H_{L}\left(s_{0}\right)=0$ (i.e., that $\mathcal{P}\left(s_{0}\right)=P_{L}$ ) and we prove that $V\left(s_{0}\right)=0$. By applying (14) to $R_{13}$, we deduce that it is divided by $\operatorname{gcd}\left(\operatorname{lc}_{t}\left(G_{1}^{*}\right), \mathrm{lc}_{t}\left(G_{3}^{*}\right)\right)=H_{L}(s)$. Thus, $H_{L}\left(s_{0}\right)=0$ implies that $R_{13}\left(s_{0}\right)=0$. In addition, by combining (5) and (24),

$$
R_{13}(s)=T(s) p_{1}(s)^{\lambda_{13}-1}=\prod_{i=1}^{n} H_{P_{i}}(s)^{m_{i}-1} V(s) p_{1}(s)^{\lambda_{13}-1}
$$

so we get that

$$
\prod_{i=1}^{n} H_{P_{i}}\left(s_{0}\right)^{m_{i}-1} V\left(s_{0}\right) p_{1}\left(s_{0}\right)^{\lambda_{13}-1}=0 .
$$


Observe that $\operatorname{gcd}\left(H_{L}, H_{P_{i}}\right)=1$ (otherwise, we would have $P_{i}=P_{L}$ ). Hence, $\prod_{i=1}^{n} H_{P_{i}}\left(s_{0}\right)^{m_{i}-1} \neq 0$. On the other hand, $\mathcal{P}\left(s_{0}\right)=P_{L}$ implies that $p_{1}\left(s_{0}\right)=$ $a_{d}$ and since we are assuming $a_{d} \neq 0$, we conclude that $V\left(s_{0}\right)=0$. Thus, we have proved that $V\left(s_{0}\right)=0$ if and only if $H_{L}\left(s_{0}\right)=0$ and, as a consequence, we have that $V(s)=H_{L}(s)^{\nu}$ for some $\nu \in \mathbb{N}$. By substituting above, we get

$$
R_{13}(s)=\prod_{i=1}^{n} H_{P_{i}}(s)^{m_{i}-1} H_{L}(s)^{\nu} p_{1}(s)^{\lambda_{13}-1} .
$$

Now, we compute $\nu$. For this purpose, we use the reparametrization

$$
\mathcal{Q}(t)=\mathcal{P}\left(\frac{\theta t}{t-1}\right)
$$

and we take $\theta$ such that $p_{1}(\theta) \neq 0$ and $\mathcal{P}(\theta) \neq P_{i}$, for $i=1, \ldots, n$. We consider the polynomials $\widetilde{G}_{i}, i=1,2,3$ and $\widetilde{R}_{i j}(i, j=1,2,3)$ introduced in (10) and (11), respectively. In addition, let $\widetilde{\delta}_{i}=\operatorname{deg}_{t}\left(\widetilde{G}_{i}\right)$ and $\widetilde{\lambda}_{i j}=$ $\min \left\{\widetilde{\delta}_{i}, \widetilde{\delta}_{j}\right\} \quad(i, j=1,2,3)$. Then, we may construct the T-function for the new parametrization, $\mathcal{Q}(t)$, as follows (see (12)):

$$
\widetilde{T}=\widetilde{R}_{13} / q_{1}^{\widetilde{\lambda}_{13}-1} .
$$

We recall that the limit point for $\mathcal{Q}(t)$ is $Q_{L}=\mathcal{P}(\theta)$ (see (8)). Since we have chosen $\theta$ such that $\mathcal{P}(\theta) \neq P_{i}$ for $i=1, \ldots, n$, we are ensuring the new limit point to be regular. Thus, we can apply Corollary 3 in [2] and we get that $\operatorname{deg}(\widetilde{T})=(d-1)(d-2)$. In addition, we have that $p_{1}(\theta) \neq 0$ and thus $\operatorname{deg}\left(q_{1}\right)=d$, which implies that $\widetilde{\delta}_{1}=\widetilde{\delta}_{3}=\widetilde{\lambda}_{13}=d$. Hence,

$$
\operatorname{deg}\left(\widetilde{R}_{13}\right)=\operatorname{deg}\left(\widetilde{T} \cdot q_{1}^{\widetilde{\lambda}_{13}-1}\right)=(d-1)(d-2)+d(d-1)=2(d-1)^{2} .
$$

Since $Q_{L}$ is a regular point, we may apply Theorem 4.1 and we get the following equality:

$$
\widetilde{R}_{13}(s)=\prod_{i=1}^{n} \widetilde{H}_{P_{i}}(s)^{m_{i}-1} \widetilde{H}_{L}(s)^{m_{L}-1} q_{1}(s)^{d-1}
$$

where $\widetilde{H}_{P_{i}}(s)$ is the fibre function of the singular point $P_{i}(i=1, \ldots, n)$ and $\widetilde{H}_{L}(s)$ is the fibre function of the singular point $P_{L}$ (note that now $P_{L}$ 
is not the limit point). All these fibre functions are computed from the parametrization $\mathcal{Q}(t)$.

Now, let us compare the expressions in (25) and (26). By computing degrees on (25), we have that

$$
\operatorname{deg}_{t}\left(R_{13}(s)\right)=\operatorname{deg}_{t}\left(\prod_{i=1}^{n} H_{P_{i}}(s)^{m_{i}-1}\right)+\operatorname{deg}_{t}\left(H_{L}(s)^{\nu}\right)+\operatorname{deg}_{t}\left(p_{1}(s)^{\lambda_{13}-1}\right) .
$$

On the other hand, from (26),

$$
\operatorname{deg}_{t}\left(\widetilde{R}_{13}(s)\right)=\operatorname{deg}_{t}\left(\prod_{i=1}^{n} \widetilde{H}_{P_{i}}(s)^{m_{i}-1}\right)+\operatorname{deg}_{t}\left(\widetilde{H}_{L}(s)^{m_{L}-1}\right)+\operatorname{deg}_{t}\left(q_{1}(s)^{d-1}\right) .
$$

We observe that $P_{i} \neq P_{L}$ and $P_{i} \neq Q_{L}$ for each $i=1, \ldots, n$ (note that $Q_{L}=\mathcal{P}(\theta)$ and we have chosen $\theta$ such that $\mathcal{P}(\theta) \neq P_{i}$ for $\left.i=1, \ldots, n\right)$. Then, by applying Corollary 2.6, we get that $\operatorname{deg}\left(H_{P_{i}}\right)=\operatorname{deg}\left(\widetilde{H}_{P_{i}}\right)=m_{i}$ for $i=1, \ldots, n$ and, therefore, $\operatorname{deg}\left(\prod_{i=1}^{n} H_{P_{i}}(s)^{m_{i}-1}\right)=\operatorname{deg}\left(\prod_{i=1}^{n} \widetilde{H}_{P_{i}}(s)^{m_{i}-1}\right)$. On the other hand, we have that $\operatorname{deg}\left(p_{1}\right)=d$ (we are assuming that $a_{d} \neq 0$ ) and $\operatorname{deg}\left(q_{1}\right)=d$ (the coefficient of $t^{d}$ in $q_{1}$ is $p(\theta)$ and we are assuming that $p(\theta) \neq 0)$. Furthermore, $a_{d} \neq 0$ implies that $d_{1}=d \geq d_{2}, d_{3}$ and thus, $\delta_{1}=\delta_{3}=\lambda_{13}=d$, so we have that $\operatorname{deg}\left(p_{1}(s)^{\lambda_{13}-1}\right)=\operatorname{deg}\left(q_{1}(s)^{d-1}\right)$.

From the above statements, we deduce that

$$
\operatorname{deg}\left(R_{13}\right)-\operatorname{deg}\left(\widetilde{R}_{13}\right)=\operatorname{deg}\left(H_{L}^{\nu}\right)-\operatorname{deg}\left(\widetilde{H}_{L}^{m_{L}-1}\right) .
$$

In addition, we know from $(9)$ that $\operatorname{deg}\left(\widetilde{H}_{L}(s)\right)$ provides the total multiplicity of $P_{L}$ (that is, $m_{L}$ ), while $\operatorname{deg}\left(H_{L}\right)$ provides just its visible multiplicity (that is, $\left.m_{L}-m_{H}\right)$. Thus, we have that

$$
\operatorname{deg}\left(R_{13}\right)=2(d-1)^{2}+\left(m_{L}-m_{H}\right) \nu-m_{L}\left(m_{L}-1\right) .
$$

In the following, we compute $\operatorname{deg}\left(R_{13}\right)$ in a different way and we compare the result obtained with $(27)$. Since we are assuming $\delta_{1}=\delta_{2}$, we may use Lema 5.1 and Remark 5.2; the last one states that

$$
\widetilde{R}_{13}(s)=(s-1)^{2(d-1)^{2}} R_{13}\left(\frac{\theta s}{s-1}\right) .
$$


By applying the change $\frac{\theta s}{s-1}=t$ to the above expression, we get

$$
\widetilde{R}_{13}\left(\frac{t}{t-\theta}\right)=\left(\frac{t}{t-\theta}-1\right)^{2(d-1)^{2}} R_{13}(t)=\left(\frac{\theta}{t-\theta}\right)^{2(d-1)^{2}} R_{13}(t)
$$

and hence,

$$
R_{13}(t)=\left(\frac{t-\theta}{\theta}\right)^{2(d-1)^{2}} \widetilde{R}_{13}\left(\frac{t}{t-\theta}\right) .
$$

Now, let us analyze the factor $\widetilde{R}_{13}\left(\frac{t}{t-\theta}\right)$. According to $(9)$, we may write $\widetilde{H}_{L}(s)$ as $\widetilde{H}_{L}(s)=(s-1)^{m_{H}} \bar{H}_{L}(s)$, where $m_{H}$ is the hidden multiplicity and $\bar{H}_{L}(s)$ is a polynomial such that $\bar{H}_{L}(1) \neq 0$. By substituting it on (26), we obtain:

$$
\begin{gathered}
\widetilde{R}_{13}(s)=\prod_{i=1}^{n} \widetilde{H}_{P_{i}}(s)^{m_{i}-1}\left((s-1)^{m_{H}} \bar{H}_{L}(s)\right)^{m_{L}-1} q_{1}(s)^{d-1}= \\
=(s-1)^{m_{H}\left(m_{L}-1\right)} \prod_{i=1}^{n} \widetilde{H}_{P_{i}}(s)^{m_{i}-1} \bar{H}_{L}(s)^{m_{L}-1} q_{1}(s)^{d-1} .
\end{gathered}
$$

Note that $\bar{H}_{L}(1) \neq 0$ and $\widetilde{H}_{P_{i}}(1) \neq 0$ for any $i=1, \ldots, n\left(\widetilde{H}_{P_{i}}(1)=0\right.$ would imply that $\left.P_{i}=\mathcal{Q}(1)=P_{L}\right)$. In addition, from (7) we have that $q_{1}(1)=a_{d} \theta^{d} \neq 0$. Thus, we may write

$$
\widetilde{R}_{13}(s)=(s-1)^{m_{H}\left(m_{L}-1\right)} \bar{R}_{13}(s),
$$

where $\bar{R}_{13}(s)$ is a polynomial such that $\bar{R}_{13}(1) \neq 0$. Hence,

$$
\widetilde{R}_{13}\left(\frac{t}{t-\theta}\right)=\left(\frac{\theta}{t-\theta}\right)^{m_{H}\left(m_{L}-1\right)} \bar{R}_{13}\left(\frac{t}{t-\theta}\right)
$$

and, by substituting in (28), up to constants, we get that

$$
\begin{aligned}
R_{13}(t)= & \left(\frac{t-\theta}{\theta}\right)^{2(d-1)^{2}}\left(\frac{\theta}{t-\theta}\right)^{m_{H}\left(m_{L}-1\right)} \bar{R}_{13}\left(\frac{t}{t-\theta}\right)= \\
& =(t-\theta)^{2(d-1)^{2}-m_{H}\left(m_{L}-1\right)} \bar{R}_{13}\left(\frac{t}{t-\theta}\right) .
\end{aligned}
$$

Therefore, we deduce that

$$
\operatorname{deg}\left(R_{13}\right)=2(d-1)^{2}-m_{H}\left(m_{L}-1\right)
$$

and, by comparing (27) and (29), we conclude that $\nu=m_{L}-1$. 
Step 2

In the following we prove that condition $\delta_{1}=\delta_{2}$ is not necessary for the theorem to be hold. Let us assume w.l.o.g. that $\delta_{1}:=\operatorname{deg}_{t}\left(G_{1}\right)>\delta_{2}:=$ $\operatorname{deg}_{t}\left(G_{2}\right)$ that is, $d=d_{1}>d_{2}, d_{3}$. Then, we consider the curve $\widehat{\mathcal{C}}$ defined by the parametrization

$$
\widehat{\mathcal{P}}(t)=\left(p_{1}(t), p_{1}(t)+\lambda p_{2}(t), p(t)\right),
$$

where $\lambda \in \mathbb{K}$. Note that we are applying a change of coordinates and then, for almost all values of $\lambda$, the curve $\widehat{\mathcal{C}}$ has the same number of singularities that $\mathcal{C}$ and they are reached at the same values of the parameter $t \in \mathbb{K}$. That is, for each singularity $P_{i}$ in $\mathcal{C}$ there exists another singularity $\widehat{P}_{i}$ in $\widehat{\mathcal{C}}$ such that $H_{P_{i}}=H_{\widehat{P}_{i}}$, and reciprocally. Furthermore, since $P_{i}$ and $\widehat{P}_{i}$ have the same fibre function their multiplicities, say $m_{i}$ and $\widehat{m}_{i}$, are the same.

The limit point for $\widehat{\mathcal{P}}(t)$ is

$$
\widehat{P}_{L}=\lim _{t \rightarrow \infty} \frac{\widehat{\mathcal{P}}(t)}{t^{d}}=\left(a_{d}: a_{d}+\lambda b_{d}: c_{d}\right)
$$

and it holds that $\widehat{\mathcal{P}}(t)=\widehat{P}_{L}$ if and only if $\mathcal{P}(t)=P_{L}$. Thus, $H_{\widehat{P}_{L}}(s)=H_{P_{L}}(s)$ and $\widehat{m}_{L}:=\operatorname{mult}_{\widehat{P}_{L}}(\widehat{\mathcal{C}})=m_{L}:=\operatorname{mult}_{P_{L}}(\mathcal{C})$.

By appropriately choosing $\lambda$ in (30), we can force the singularities of the curve to keep their ordinary character. In addition, we can get that $a_{d}+\lambda b_{d} \neq 0$, which ensures that $\operatorname{deg}\left(p_{1}(t)+\lambda p_{2}(t)\right)=\max \left\{d_{1}, d_{2}\right\}=d_{1}=d$. Now, for each $i=1,2,3$, let $\widehat{G}_{i}$ be the equivalent polynomial to $G_{i}$ but computed from $\widehat{\mathcal{P}}(t)$. Observe that $\widehat{G}_{1}=G_{1}$ and

$$
\widehat{G}_{2}(s, t)=\left(p_{1}(s)+\lambda p_{2}(s)\right) p(t)-p(s)\left(p_{1}(t)+\lambda p_{2}(t)\right)
$$

which implies that $\operatorname{deg}_{t}\left(\widehat{G}_{1}\right)=\operatorname{deg}_{t}\left(\widehat{G}_{2}\right)=d$. Then, the conditions imposed in Step 1 are satisfied and the theorem holds for $\widehat{\mathcal{P}}(t)$. Thus,

$$
\widehat{R}_{12}(s):=\operatorname{Res}_{t}\left(\frac{\widehat{G}_{1}}{t-s}, \frac{\widehat{G}_{2}}{t-s}\right)=p(s)^{d-1}\left(\prod_{i=1}^{n} H_{\widehat{P}_{i}}(s)^{\widehat{m}_{i}-1}\right) H_{\widehat{P}_{L}}(s)^{\widehat{m}_{L}-1},
$$

where $\widehat{P}_{1}, \ldots, \widehat{P}_{n}$ and $\widehat{P}_{L}$ are the singularities of $\widehat{\mathcal{C}}$, and $\widehat{m}_{1}, \ldots, \widehat{m}_{n}, \widehat{m}_{L}$ their respective multiplicities. On the other hand, we have remarked above that 
the singularities of $\mathcal{C}$ and $\widehat{\mathcal{C}}$ (and also their multiplicities) are the same. Then, we have that

$$
\widehat{R}_{12}(s)=p(s)^{d-1} \prod_{i=1}^{n} H_{P_{i}}(s)^{m_{i}-1} H_{L}(s)^{m_{L}-1} .
$$

Now we observe that

$$
\begin{gathered}
\widehat{G}_{2}(s, t)=\left(p_{1}(s)+\lambda p_{2}(s)\right) p(t)-p(s)\left(p_{1}(t)+\lambda p_{2}(t)\right)= \\
p_{1}(s) p(t)-p(s) p_{1}(t)+\lambda\left(p_{2}(s) p(t)-p(s) p_{2}(t)\right)=\left(G_{1}+\lambda G_{2}\right)(s, t) .
\end{gathered}
$$

Thus, by applying some well known properties of the resultants (see e.g. Appendix B in [16]), we get that

$$
\begin{gathered}
\widehat{R}_{12}(s)=\operatorname{Res}_{t}\left(\frac{G_{1}}{t-s}, \frac{G_{1}+G_{2}}{t-s}\right)= \\
=\mathrm{lc}_{t}\left(\frac{G_{1}}{t-s}\right)^{d-1} \prod_{i=1}^{d-1}\left(\frac{G_{1}\left(s, \alpha_{i}(s)\right)+G_{2}\left(s, \alpha_{i}(s)\right)}{\alpha_{i}(s)-s}\right)
\end{gathered}
$$

where $\alpha_{1}(s), \ldots, \alpha_{d-1}(s)$ are the $d-1$ roots of the polynomial $G_{1}^{*}(s, t):=$ $G_{1}(s, t) /(t-s) \in(\mathbb{K}[s])[t]$. That is, for each $i=1, \ldots, d-1$, we have that $\alpha_{i}(s) \neq s$ and $G_{1}\left(s, \alpha_{i}(s)\right)=0$. Thus, the last formula may be written as

$$
\widehat{R}_{12}(s)=\mathrm{lc}_{t}\left(\frac{G_{1}}{t-s}\right)^{d-\delta_{2}} \mathrm{lc}_{t}\left(\frac{G_{1}}{t-s}\right)^{\delta_{2}-1} \prod_{i=1}^{d-1}\left(\frac{G_{2}\left(s, \alpha_{i}(s)\right)}{\alpha_{i}(s)-s}\right),
$$

and, hence,

$$
\widehat{R}_{12}(s)=\operatorname{lc}_{t}\left(\frac{G_{1}}{t-s}\right)^{d-\delta_{2}} \operatorname{Res}_{t}\left(\frac{G_{1}}{t-s}, \frac{G_{2}}{t-s}\right) .
$$

Note that $\mathrm{lc}_{t}\left(\frac{G_{1}}{t-s}\right)=\mathrm{lc}_{t}\left(G_{1}\right)=p(s)$ since $d_{3}<d_{1}$, so we deduce that

$$
\widehat{R}_{12}(s)=p(s)^{d-\delta_{2}} \operatorname{Res}_{t}\left(\frac{G_{1}}{t-s}, \frac{G_{2}}{t-s}\right)=p(s)^{d-\delta_{2}} R_{12}(s) .
$$

Finally, by combining (31) and (32), we conclude that

$$
R_{12}(s)=p(s)^{\delta_{2}-1} \prod_{i=1}^{n} H_{P_{i}}(s)^{m_{i}-1} H_{L}(s)^{m_{L}-1} .
$$

The result follows from the assumption that $\delta_{2}=\min \left\{\delta_{1}, \delta_{2}\right\}=\lambda_{12}$. 


\section{Acknowledgements}

This work has been partially funded by Ministerio de Economía y Com-

petitividad under the Project MTM2017-88796-P. The second author belongs to the Research Group ASYNACS (Ref. CCEE2011/R34).

[1] Abhyankar, S.S., (1990). Algebraic Geometry for Scientists and Engineers. Math. Surveys Monogr., vol. 35, American Mathematical Society, Providence, RI.

[2] Blasco, A., Pérez-Díaz, S. (2017). Resultants and Singularities of Parametric Curves. See arXiv:1706.08430v4 [math.AG].

[3] Busé, L., D'Andrea, C. (2012) Singular factors of rational plane curves. Journal of Algebra, 357, 322-346.

[4] Chen, F., Wang, W., Liu, Y. (2008) Computing singular points of plane rational curves. J. Symbolic Comput. 43 (2) 92-117.

[5] Gutierrez, J., Rubio, R., Yu, J.-T. (2002). Resultant for rational functions. Proceedings of the American Mathematical Society. Vol. 130 (8). pp. 2237-2246.

[6] Harris, J. (1995). Algebraic Geometry. A first Course. Springer-Verlag.

[7] Hoffmann, C.M., Sendra, J.R., Winkler, F. (1997). Parametric Algebraic Curves and Applications. J. Symbolic Computation. Vol. 23.

[8] Hoschek, J., Lasser, D. (1993). Fundamentals of Computer Aided Geometric Design. A.K. Peters Wellesley MA., Ltd.

[9] Jüttler, B., Piene, R. (2007). Geometric Modeling and Algebraic Geometry. Springer Science and Business Media, 2007.

[10] Park, H. (2002). Effective computation of singularities of parametric affine curves. Journal of Pure and Applied Algebra. Vol. 173, pp. 49-58.

[11] Pérez-Díaz, S. (2006). On the problem of proper reparametrization for rational curves and surfaces. Computer Aided Geometric Design. Vol. 23(4). pp. 307-323. 
[12] Pérez-Díaz, S. (2007). Computation of the Singularities of Parametric Plane Curves. Journal of Symbolic Computation. Vol. 42/8. pp. 835-857.

[13] Pérez-Díaz, S., Sendra, J.R., Villarino, C. (2015). Computing the Singularities of Rational Surfaces. Mathematics of Computation. Vol. 84, N. 294, pp. 1991-2021.

[14] Rubio, R., Serradilla, J.M., Vlez, M.P. (2009). Detecting real singularities of a space curve from a real rational parametrization. Journal of Symbolic Computation. Vol. 44, Issue 5, pp. 490-498.

[15] Sederberg, T., Goldman, R., Du, H. (1997). Implicitizing Rational Curves by the Method of Moving Algebraic Curves. Journal of Symbolic Computation. Vol. 23, pp. 153-175.

[16] Sendra, J.R., Winkler, F., Pérez-Díaz, S. (2007). Rational Algebraic Curves: A Computer Algebra Approach. Series: Algorithms and Computation in Mathematics. Vol. 22. Springer Verlag.

[17] Shafarevich, I.R., (1994). Basic algebraic geometry Schemes; 1 Varieties in projective space. Berlin New York: Springer-Verlag.Vol. 1.

[18] Shi, X., Jia, X., Goldman, R. (2013). Using a bihomogeneous resultant to find the singularities of rational space curves. Journal of Symbolic Computation. Volume 53. pp. 1-25.

[19] Song, N., Chen, F., Goldman, R. (2007). Axial moving lines and singularities of rational planar curves. Comput. Aided Geom. Design. Vol. 24(4). pp. 200-209.

[20] Van den Essen, A., Yu, J.-T. (1997). The D-resultant, singularities and the degree of unfaithfulness. Proceedings of the American Mathematical Society. Vol. 125 (3), pp. 689-695. 